\title{
Philosophiques
}

\section{Typologie des tendances théoriques du féminisme contemporain}

\section{Guy Bouchard}

Volume 18, numéro 1, printemps 1991

URI : https://id.erudit.org/iderudit/027143ar

DOI : https://doi.org/10.7202/027143ar

Aller au sommaire du numéro

Éditeur(s)

Société de philosophie du Québec

ISSN

0316-2923 (imprimé)

1492-1391 (numérique)

Découvrir la revue

Citer cet article

Bouchard, G. (1991). Typologie des tendances théoriques du féminisme

contemporain. Philosophiques, 18(1), 119-167. https://doi.org/10.7202/027143ar
Résumé de l'article

L'étude des rapports entre féminisme et philosophie politique présuppose une classification des principales tendances théoriques du féminisme contemporain. Nous présentons d'abord la typologie proposée par Alison Jaggar et nous indiquons les problèmes qu'elle pose. Nous examinons ensuite un ensemble de textes consacrés, expressément ou non, à la taxonomie de ces tendances, pour dégager une grille permettant de les rassembler dans un même cadre théorique explicitant les enjeux qu'elles thématisent, mais aussi ceux qui sont ignorés. 


\title{
TYPOLOGIE DES TENDANCES THÉORIQUES DU FÉMINISME CONTEMPORAIN ${ }^{1}$
}

\author{
par Guy Bouchard
}

\begin{abstract}
RÉsumé. L'étude des rapports entre féminisme et philosophie politique présuppose une classification des principales tendances théoriques du féminisme contemporain. Nous présentons d'abord la typologie proposée par Alison Jaggar et nous indiquons les problèmes qu'elle pose. Nous examinons ensuite un ensemble de textes consacrés, expressément ou non, à la taxonomie de ces tendances, pour dégager une grille permettant de les rassembler dans un même cadre théorique explicitant les enjeux qu'elles thématisent, mais aussi ceux qui sont ignorés.
\end{abstract}

ABSTRACT. The study of the relation between feminism and political philosophy presupposes a classification of the main theoretical trends of contemporary feminism. The paper first presents Alison Jaggar's taxonomy and underlines its problems. This is followed by the survey of a set of studies dealing, expressly or not, with the taxonomy of these trends. The analysis of these and of Jaggar's studies leads to a grid which locates the trends in the same theoretical framework, shows the stakes that they tackle and reveals those that are not discussed.

1. Texte produit dans le cadre d'un projet subventionné par le Conseil de Recherches en Sciences Humaines du Canada, et consacré à la Pbilosopbie bétéropolitique du féminisme. 
"La plus grande partie de la philosophie politique ne tient nul compte des femmes, écrivait naguère Ti-Grace Atkinson (1975:47), et, en raison même de cette omission, c'est une philosophie dont on ne peut tenir aucun compte. * Mais si le féminisme pouvait se passer de la philosophie politique, peut-être celle-ci ne peut-elle plus faire l'économie du féminisme? Comme le souligne Susan Moller Okin (1979:5-10), si la grande tradition de la philosophie politique se compose en général d'écrits par les hommes, pour les hommes et au sujet des hommes, il ne suffit pas d'inclure les femmes dans le champ de ses préoccupations parce que le présupposé de l'inégalité entre les sexes corrompt d'emblée cette éventuelle structure d'accueil. Les fondements mêmes de la philosophie politique sont donc à repenser, si l'on veut qu'elle cesse de jouer le rôle d'une idéologie androcentrique cautionnant la subordination des femmes. Comme le souligne Alison Jaggar (1983 :7), les théoriciennes féministes explorent la possibilité d'appliquer les catégories politiques existantes à des domaines que jusqu'ici l'on croyait au-delà de la sphère politique: les réflexions sur l'égalité des femmes ne se bornent pas à réclamer des chances égales et un traitement préférentiel sur le marché du travail, elles se demandent aussi s'il faut, par exemple, instaurer des congés de maternité payés ou promouvoir les bébés éprouvettes, et l'étude de ces nouveaux problèmes, en manifestant les limites de la philosophie politique traditionnelle, contribue à sa transformation.

"Le" féminisme n'est toutefois pas une entité homogène. Fondé sur la conviction que la situation des femmes dans la société est injuste et doit en conséquence être changée ${ }^{2}$, il se fragmente non seulement sur le plan théorique, en fonction des diverses conceptions de l'oppression

2. Cf. par exemple Atkinson (1975:116): «Tous les groupes féministes que je connais acceptent au moins un point, à savoir que les femmes sont au moins les victimes de la discrimination *. Et Jaggar (1981:5) : * Feminists are united by a belief that the unequal and inferior social status of women is unjust and needs to be changed . Ces propos d'Atkinson et de Jaggar ne constituent pas des exemples de définitions formelles du féminisme, mais plutor des indices de la pertinence de la définition minimale que nous présentons comme une «conviction * généralement partagée. Le problème d'une véritable définition mériterait une étude spéciale, mais celle-ci aurait avantage à tenir compte de la diversité dont nous tentons d'esquisser les contours. Sans doute pourrait-on objecter que formuler cette conviction en termes de justice laisse entendre que le féminisme disparaîtrait avec l'injustice de la situation des femmes, mais cette inquiétude n'est-elle pas du meme ordre que celle qui se demanderait ce qu'il adviendra des mouvements révolutionnaires après le succès de la révolution? Le féminisme pourrait disparaitre, mais il pourrait aussi se donner une nouvelle vocation : le jour où il ne restera que ce problème à résoudre, on pourra en rediscuter. 
et de la manière d'y mettre fin, mais aussi sur le plan empirique, en fonction de la dispersion temporelle et géographique des groupes qui s'en réclament. L'un des problèmes majeurs liés à toute tentative d'articuler philosophie politique traditionnelle et féminisme consiste donc à prendre la mesure de cette fragmentation avant d'en tirer les conséquences. C'est à ce problème que sera consacrée la présente étude. Si souhaitable que soit en effet une réarticulation de la philosophie politique tenant compte des problèmes nouveaux soulevés par le féminisme, il est d'autant moins possible de l'esquisser dans le cadre d'un article qu'elle nous semble présupposer un travail moins glorieux mais pourtant nécessaire : la mise en place d'une typologie des tendances théoriques du féminisme contemporain.

Un tel travail a pour objectif premier d'élaborer un cadre permettant d'intégrer les diverses images que le féminisme donne de lui-même, mais en adoptant un point de vue spécifique, celui du rapport de chaque tendance à la transformation de la société. Ces images, nous les empruntons non seulement à des ouvrages qui abordent eux-mêmes le problème de la typologie, mais aussi à des textes polémiques promouvant une orientation en la distinguant des autres, ainsi qu'à des études précisant le sens de certaines orientations. Nous nous en tiendrons à des sources francophones et anglophones, mais sans limiter la typologie à un point de vue national, par exemple celui du féminisme français ou celui du féminisme américain, d'une part parce que les tendances, dans la mesure où elles sont théoriques, ne sont pas bornées par les frontières géographiques, d'autre part parce qu'une telle restriction nous obligerait à sacrifier inutilement une importante partie de notre documentation; dans la documentation francophone en effet, si certains textes se préoccupent exclusivement du féminisme français (Beauvoir 1984, Garcia Guadilla 1981), d'autres se consacrent au féminisme américain (Ballorain 1972, Castro 1984, Le Calvez 1979), au féminisme francophone et au féminisme anglophone (Descarries-Bélanger et Roy 1988), voire même au féminisme international (Michel 1980); inversement, outre les textes anglophones qui s'en tiennent à la perspective américaine, d'autres se préoccupent exclusivement du féminisme français (Marks et De Courtivron 1980) et d'autres encore débordent la perspective nationale (Donovan 1985, Jaggar 1983, McFadden 1984, Rowbotham 1973). 
La première partie du texte examinera la tentative la plus approfondie de rendre compte des tendances du féminisme, celle d'Alison Jaggar. Cette tentative compare la philosophie politique du féminisme libéral, du féminisme marxiste, du féminisme radical et du féminisme socialiste, elle réalise donc l'articulation entre féminisme et philosophie politique au seuil de laquelle s'arrêtera notre démarche, mais nous montrerons que cette articulation est problématique précisément parce que ce qu'elle articule est déjà problématique au niveau de la typologie. La méthode de l'analyse combinatoire, en dégageant les éléments pertinents des descriptions proposées par Jaggar et en les recombinant de façon à faire jaillir toutes les possibilités qu'ils recèlent, nous permettra alors de suggérer une première grille des tendances féministes effectives ou possibles. Consacrée à une documentation polyphonique, la seconde partie mettra en scène un réseau complexe de caractérisations des tendances féministes qui manifestera le caractère spécifique de l'option privilégiée par Jaggar et nous suggérera de nouveaux éléments de base à in tégrer à notre grille. La troisième partie reformulera celle-ci en tenant compte de ces éléments et en discutant les problèmes soulevés par la démarche antérieure.

\section{Alison Jaggar et les tendances du féminisme}

Jaggar a abordé à deux reprises le problème de la présentation des tendances du féminisme. Nous décrirons séparément ces deux présentations afin de pouvoir tirer profit de leur comparaison.

\subsection{Version initiale}

L'article "Political Philosophies of Women's Liberation * (Jaggar 1981), publié pour la première fois en 1977, reprend une communication prononcée dans un congrès de philosophie en 1972 : il n'est pas indifférent de tenir compte de cette date car, si l'on admet que la seconde vague du féminisme américain devient un mouvement social avec la fondation de la National Organization for Women (N.O.W.) en $1963^{3}$, et si l'on prend conscience de l'extraordinaire proli-

3. Cf. Ballorain (1972:66) : „Betty Friedan, les historiens du mouvement, la plupart des féministes, s'accordent maintenant pour considérer N.O.W. comme le premier groupe du Women's Movement, voire du Women's Liberation Movement, le plus puissant, le plus efficace, le mieux organisé.. 
fération de groupes et groupuscules féministes à partir de la sécession du premier noyau radical en $1968^{4}$, il faut bien reconnaître que toute entreprise visant à dégager quelques orientations majeures au sein de cette mouvance requérait lucidité, culture et courage.

Si le féminisme dénonce l'injustice de la position sociale des femmes, il s'oppose par définition à la conception conservatrice. Celle-ci, même si elle admet que certaines femmes sont défavorisées, considère que leurs épreuves ne font pas partie d'une oppression sociale systématique des femmes et que celles-ci, en tant que groupe, ne sont pas victimes d'une injustice. Les différences évidentes dans les rôles sociaux des deux sexes sont alors rationalisées, soit en prétendant que le rôle féminin n'est pas inférieur au rôle masculin, ce qui aboutit à une sorte d'apartheid sexual ${ }^{5}$ de l'égalité dans la complémentarité; soit en soutenant que la femme est par essence mieux adaptée au rôle sexual féminin traditionnel, ce qui présuppose une inégalité sexuale naturelle. Dans un cas comme dans l'autre, la conception conservatrice présuppose des différences innées entre hommes et femmes, d'où découlent des fonctions sociales hétérogènes que la loi devrait faire respecter si l'opinion et la coutume n'y suffisent pas.

Bien qu'elles s'opposent toutes à cette conception, les tendances majeures du féminisme ne s'entendent pas sur les changements sociaux requis, et leurs divergences s'enracinent, au-delà des questions de stratégie et de tactique, dans une vision différente de la nature des intérêts et de la libération des femmes. Jaggar distingue trois orientations majeures : le féminisme libéral, le féminisme marxiste classique et le féminisme radical, - ainsi que deux « directions nouvelles»: le séparatisme lesbien et le féminisme socialiste.

La tradition libérale, dont on trouve l'expression classique dans un ouvrage comme celui de John Stuart Mill sur L'asservissement des femmes, anime aujourd'hui des groupes comme la N.0.W., qui promeut l'amélioration du

4. Pour un témoignage personnel sur les motils de cette scission : Ackinson 1975 : 25-27, 57. Pour une description de la prolifération des groupes féministes entre 1968 et 1972 : Ballorain 1972.

5. J'emploie l'adjectif « sexual * pour désigner tout ce qui a trait à la différence entre les sexes sans connotation strictement sexuelle. Cela permet par exemple de parler d'identicé sexuale, plutor que de * genre *, en référence aux stéréotypes masculins et féminins, et d'appeler sexualisation le processus par lequel ces stéréotypes sont acquis. 
statut des femmes grâce à des réformes légales. Le féminisme libéral présuppose une foi profonde en l'autonomie de la personne, l'intervention de l'État dans les affaires personnelles devant être réduite au minimum, ainsi que la croyance que la justice requiert que les critères permettant à une personne d'accomplir une fonction sociale soient fondés sur sa seule capacité à l'accomplir plutôt que sur des facteurs comme le sexe, la race ou la religion. Dans ce contexte, la libération de la femme est liée à la suppression des entraves à son entrée dans la sphère publique, et donc à sa possibilité de déterminer son rôle social aussi librement que les hommes dans la mesure où l'État lui assure l'égalité des chances pour que la compétition devienne équitable. Une telle libération n'affecte pas l'ensemble de la structure sociale et, si elle enlève aux hommes leur position privilégiée, elle les déleste en même temps de l'entière responsabilité de supporter leur famille et de défendre le pays, tout en leur demandant de contribuer sur une base égalitaire au travail domestique.

Pour le féminisme marxiste classique par contre, l'individu n'est pas d'abord préoccupé par la maximisation de ses intérêts personnels : la nature humaine, flexible, étant le reflet de l'organisation économique de la société, il faut rééduquer les gens pour leur apprendre qu'ils ont des buts et intérêts communs plutôt que compétitifs. La liberté, pour la femme, n'est pas absence de discrimination à son égard, mais libération de la coercition exercée par la nécessité économique. L'égalité ne se réduit pas à l'équilibration des chances dans un univers de compétition, elle est égalité approximative de satisfaction des besoins matériels. Et l'État, avant sa disparition, ne se contentera pas d'établir des règles équitables de compétition : détenteur des moyens de production, il assumera la responsabilité économique et supprimera ainsi le fondement de la dépendance de la femme à l'égard de l'homme. Car l'oppression des femmes, selon le marxisme classique, est liée à la propriété privée, même si celle-ci ne l'a pas créée, et disparaîtra avec elle. Le féminisme fait ainsi partie du combat plus global pour l'avènement de la société communiste et les intérêts à long terme des femmes sont ceux de la classe laborieuse. Quant à leur oppression spécifique, qui découle de leur exclusion du circuit de la production publique et de leur confinement à des tâches domestiques privées, elle sera abolie par leur intégration à l'industrie publique, qui mettra fin à leur dépendance économique, et par la socialisation du travail domestique, le mariage monogamique ne reposant plus dès 
lors que sur l'inclination mutuelle d'êtres libres et égaux. Les hommes bénéficieront eux aussi de cette libération de la femme, puisqu'ils seront délivrés de l'oppression de classe et de la responsabilité de pourvoir aux besoins de la famille.

Le féminisme radical, tel que l'expriment par exemple Ti-Grace Atkinson et Shulamith Firestone ${ }^{6}$, nie à la fois la thèse libérale que la base de l'oppression des femmes réside dans un manque de droits politiques ou civils, et la thèse marxiste qu'elle résulte de la division de la société en classes. Pour les radicales, et cela peut sembler une régression au conservatisme, les racines de l'oppression sont biologiques ${ }^{7}$ : la faiblesse liée au fait de porter des enfants a rendu les femmes dépendantes des hommes pour leur survie physique. Or l'asservissement de la femme par l'homme constitue la forme de base de l'oppression, antérieure à l'institution de la propriété privée et de la domination de classe; par suite, les relations de pouvoir dans la famille biologique deviennent le modèle de la compréhension des autres formes d'oppression, et il faut combattre le sexisme avant de combattre le racisme et le capitalisme. Dans cette perspective, la libération des femmes passe par une révolution biologique qui, grâce aux progrès de la technologie, les délivrera de cette inégalité fondamentale qu'est la tâche de porter des enfants : avec la reproduction artificielle, porter les enfants et les élever deviendront en effet des devoirs incombant à la société tout entière, ce qui rendra possible l'autodétermination des femmes et des enfants et leur intégration à tous les aspects de la vie sociale. Si la technologie supprime la nécessité du travail, les femmes, en effet, ne seront plus économiquement dépendantes des hommes. Et si elle supprime les bases de la famille, cela éliminera les rôles sociaux ainsi que la répression sexuelle, et permettra l'instauration d'un cadre politique anarchiste. Les hommes

6. La description de cette tendance se fonde surtout sur les idées de Firestone (1972)

7. L'aspect conservateur de cette thèse tient à son recours à une explication de type biologique. Mais les conservatrices n'admettent Evidemment pas que la biologie est un facteur d'oppression, au contraire elle justifie pour elles la "différence complémentaire *. Cette divergence profonde entre les radicales et les conservatrices est malheureusement occultée dans la présencation de Jane English (1981 : 2), qui formule les choses comme si les conservatrices dénonçaient elles aussi l'oppression des femmes et réclamaient leur libération : «Alison Jaggar describes and compares six major positions on women's liberation. They desagree not merely about the means that should be used, but about what equality and justice call for. Radical feminists, following Shulamith Firestone, agree with the conservatives that the source of women's oppression is fundamentally biological . . Le caractère non féministe de la position conservatrice est reconnu dans l'anthologie de Jaggar et Struhl (1978: XII) 
bénéficieront eux aussi de cette transformation car, débarrassés du rôle de pourvoyeurs, ils pourront participer sur une base égalitaire à la production et à l'éducation des enfants.

En plus de ces trois tendances, il faut tenir compte de deux orientations nouvelles. Le séparatisme lesbien est un mouvement en émergence selon lequel les femmes devraient s'abstenir, provisoirement ou non, des rapports hétérosexuels, car ceux-ci contribuent à la perpétuation de la suprématie masculine; parmi les séparatistes, les unes adoptent une approche méthodologique marxiste au sens large, tandis que d'autres, plus radicales, réclament une société matriarcale qui exclurait probablement la participaton à part entière des hommes. Quant au féminisme socialiste, il se constitue en excluant les déficiences du féminisme marxiste classique comme du féminisme radical, et en intégrant leurs intuitions valables dans une nouvelle théorie. Ainsi, l'on rejette comme anhistorique, antidialectique et utopique la thèse radicale que la libération des femmes requiert l'abolition de la maternité, et si l'on admet avec le marxisme que le socialisme constitue la principale précondition à la libération des femmes, on ajoute qu'elle n'est pas suffisante parce que le sexisme peut continuer à sévir malgré la propriété publique des moyens de production. II faut donc conjuguer le développement d'une conscience féministe spécifique et la modification de la base économique, ce qui implique une transformation totale de la société. Entretemps, les féministes socialistes croient qu'il est possible de commencer à changer les rapports de pouvoir dans les relations entre hommes et femmes et, rejetant le déterminisme économique rigide, elles se montrent sympathiques envers les tentatives individuelles de modifier les styles de vie et d'instaurer le partage des responsabilités même si ces tentatives ne peuvent pas réussir pleinement dans un contexte capitaliste.

Jaggar n'est pas certaine que ces deux orientations soient des idéologies nouvelles plutôt que des extensions d'idéologies existantes. Mais la version matriarcophile du séparatisme lesbien est assurément originale par son interprétation de la liberté comme liberté à l'égard de l'homme et par sa suggestion que la femme est supérieure à l'homme de façon innée. Quant au féminisme socialiste, il est sans doute une extension du féminisme marxiste classique, dont il partage les conceptions de la nature humaine, de la liberté, de l'égalité et du rôle de l'État, mais en soulignant que 
la situation des femmes et la sphère des relations personnelles devraient être soumises à une analyse plus attentive.

Le seul principe clairement énoncé sur lequel se fonde cette typologie stipule que le désaccord sur les changements requis découle de divergences relatives à la nature des intérêts et aux formes de la libération des femmes. L'explicitation de ces divergences repose sur la description des thèses majeures de chacune des tendances observées. Nous allons maintenant tenter de dégager de ces descriptions quelques sèmes ou critères distinctifs permettant de mieux situer les tendances en dégageant leurs enjeux majeurs.

L'univers discursif initial est celui des diverses conceptions de la femme dans la société. Cet univers se compose de deux sous-ensembles, celui des conceptions conservatrices et celui des conceptions féministes : le premier soutient que la situation des femmes en tant que groupe n'est pas injuste et qu'en conséquence la société n'a pas à être transformée, tandis que pour le second la position sociale des femmes est injuste et requiert une métamorphose de la société. C'est évidemment dans le cadre de ce dernier que les tendances du féminisme s'inscrivent.

Si le féminisme se définit par la conviction que la situation sociale des femmes est injuste et doit être modifiée, on peut prévoir que ses diverses tendances se caractériseront par leur façon d'analyser l'oppression et par leurs propositions de libération, c'est-à-dire de transformation de la société.

Une première distinction entre transformation partielle et transformation globale permet d'opposer le féminisme réformateur au féminisme révolutionnaire. Le premier correspond, dans les descriptions de Jaggar, au féminisme libéral, pour lequel l'oppression des femmes, fondée sur leur sexe, peut être contrée par des réformes légales destinées à éliminer ce qui entrave leur accès à la sphère publique en leur conférant des droits qui devraient leur donner l'égalité des chances. Quant au féminisme révolutionnaire, il regroupe toutes les autres tendances.

Essayons d'abord de départager celles-ci en termes de cause de l'oppression. Selon que l'on assimile cette cause à la classe sociale ou au sexe des individus, on peut prévoir trois tendances : l'une mettant l'accent sur la classe, l'autre sur le sexe, et la troisième, sur les deux facteurs simultanément. Le premier cas correspond au féminisme marxiste classique : l'oppression des femmes se situe dans le prolon- 
gement de l'exploitation du prolétariat par le capitalisme, bien qu'elle soit renforcée de façon spécifique par l'exclusion des femmes du circuit de la production, et elle disparaîtra avec l'avènement de la société communiste et la réintégration des femmes dans la sphère publique grâce à la socialisation du travail domestique. Le second cas est illustré par le féminisme radical : l'oppression des femmes est due à leur sexe, plus précisément à la faiblesse physique engendrée par la maternité, et elle disparaîtra quand la technologie permettra aux femmes de se libérer de celle-ci en la transformant en responsabilité collective; et comme l'oppression sexuale est source et modèle des autres formes d'oppression, sa suppression posera les fondements de leur élimination. Le troisième cas devrait correspondre au féminisme socialiste. Voilà en effet ce que suggère l'affirmation que ce dernier résulte de l'exclusion des déficiences et de l'assomption des thèses valables des féminismes marxiste et radical. Mais voilà pourtant ce que dément l'idée qu'il ne serait qu'une extension du féminisme marxiste classique. Démenti injustifié: si l'avènement d'une société communiste ne met pas nécessairement fin au sexisme, cela signifie que l'oppression des femmes n'est pas uniquement un phénomène de classe et qu'elle ne peut pas être résolue par la seule dissolution des classes. Ces tergiversations peuventelles s'expliquer par l'état embryonnaire du féminisme socialiste à l'époque où le texte de Jaggar a été rédigé, et par une présentation unilatérale du féminisme radical mettant surtout l'accent sur la contribution de Shulamith Firestone? Dans ce contexte, le féminisme socialiste peut bien retenir l'oppression de classe tout en dénonçant ses limites, mais il ne peut pas y joindre l'oppression sexuale telle qu'elle s'exprime dans le féminisme radical parce qu'il n'est pas prêt à l'interpréter en termes de faiblesse due à la maternité.

Quant au séparatisme lesbien, nous n'en retiendrons que la version considérée comme une orientation originale par l'auteure, celle qui prône une société matriarcale excluant probablement la participation des hommes. Dans la mesure où cette orientation dénonce l'oppression des femmes en tant que telles, et non en tant que membres d'une classe économique, elle s'apparente au féminisme radical. Nous considérerons dès lors que celui-ci comporte deux variantes, l'une séparatiste et l'autre non séparatiste. En résumé : 


\begin{tabular}{|c|c|c|c|c|c|c|}
\hline & \multirow{3}{*}{$\begin{array}{l}\text { Transformation } \\
\text { partielle de la } \\
\text { société: } \\
\text { oppression } \\
\text { fondée sur } \\
\text { le sexe et } \\
\text { liberation non } \\
\text { séparatiste }\end{array}$} & \multicolumn{5}{|c|}{$\begin{array}{l}\text { transformation globale de } \\
\text { la société }\end{array}$} \\
\hline & & \multicolumn{3}{|c|}{ oppression } & \multicolumn{2}{|c|}{ libération } \\
\hline & & sexe & classe & $\begin{array}{l}\text { sexe et } \\
\text { classe }\end{array}$ & $\begin{array}{l}\text { non } \\
\text { séparatiste }\end{array}$ & separatiste \\
\hline $\begin{array}{l}\text { féminisme } \\
\text { libera! }\end{array}$ & + & & & & & \\
\hline $\begin{array}{l}\text { féminisme } \\
\text { radical }\end{array}$ & & + & & & + & \\
\hline $\begin{array}{l}\text { féminisme } \\
\text { séparatiste } \\
\text { lesbien }\end{array}$ & & + & & & & + \\
\hline $\begin{array}{l}\text { féminisme } \\
\text { manxiste }\end{array}$ & & & + & & + & \\
\hline $\begin{array}{l}\text { féminisme } \\
\text { socialiste }\end{array}$ & & & & + & + & \\
\hline
\end{tabular}

Voyons maintenant si cette réarticulation des tendances du féminisme correspond à ce que nous propose Jaggar, dans sa...

\subsection{Version finale}

Feminist Politics and Human Nature (Jaggar 1983) est un ouvrage remarquable qui, après une esquisse des rapports entre féminisme et philosophie politique, étudie les positions respectives des quatre tendances majeures de ce premier à l'égard des théories de la nature humaine et de la politique, puis des théories de la connaissance. Bien qu'elle soit beaucoup plus détaillée et nuancée, nous ne reprendrons pas la description de ces tendances, si ce n'est dans la mesure où certains éléments en seront pertinents à la discussion des principes sur lesquels repose la typologie de Jaggar ${ }^{8}$.

Le but que se propose l'auteure est d'évaluer les tendances du féminisme en clarifiant leurs énoncés, présupposés et implications. Or ces présupposés sont liés à quatre conceptions différentes et incompatibles de la nature

8. Ces principes sont énoncés au tout début de l'ouvrage (Jaggar 1983:8-13). L'incompatibilité des tendances est réaffirmée à la fin (p. 353). 
humaine, d'où procèdent quatre théories politiques systématiques de la libération des femmes : le féminisme libéral, le féminisme marxiste traditionnel, le féminisme radical et le féminisme socialiste. Les théories differrent également par leurs analyses de l'oppression, selon que les femmes sont considérées comme opprimées par les hommes; ou les hommes et les femmes, par le système des rôles sexuaux; ou selon que l'oppression est présentée comme un résultat du capitalisme. L'étude de chaque tendance comporte trois mouvements: identifier sa conception de la nature humaine, puis tisser les liens entre celle-ci et la critique de la société contemporaine ainsi que les propositions de changement social offertes par chaque théorie, et enfin un exposé des critères épistémologiques permettant à chacune de s'autojustifier. Comme ces tendances sont en constante évolution, il est possible que leur description ne soit que provisoire. Et comme l'analyse dépend toujours du but poursuivi et que le but de l'auteure n'est pas d'effectuer une taxinomie du plus grand nombre de sous-espèces possible, mais d'élaborer une méthodologie valable susceptible de servir de base à la théorie et à la pratique de la libération des femmes, la typologie s'oriente vers la réduction, plutôt que vers la prolifération des théories, elle s'en tient aux genres, aux façons foncièrement incompatibles de conceptualiser la situation des femmes. Les quatre tendances retenues, qui constituent des types idéaux minimisant les similitudes et accentuant les différences, sont considérées par l'auteure comme les plus plausibles parmi celles qui existent. Et la plus plausible d'entre elles serait le féminisme socialiste.

La typologie de Jaggar repose donc sur deux critères : celui de la plausibilité et celui de la systématicité, c'est-à-dire de la corrélation entre conception de la nature humaine, analyse de l'oppression et vision de la libération. Notre discussion ne contestera pas, du moins de prime abord, la pertinence de ces critères, elle se contentera de montrer que leur application est doublement problématique, tant dans ses inclusions que dans ses exclusions.

Les problèmes d'inclusion concernent le féminisme radical et le féminisme socialiste.

En vertu du critère de systématicité, une tendance féministe se caractérise par une conception irréductible de la nature humaine, par une analyse de l'oppression des femmes et par une vision de leur libération. Or «le * fémi- 
nisme radical ${ }^{9}$ ne correspond pas à ces caractéristiques : il n'a pas de conception unifiée de la nature humaine, il offre des analyses différentes de l'oppression et diverses visions de la libération.

Jaggar présente quatre conceptions radicales de la nature humaine ${ }^{10}$. Pour le féminisme radical androgynophile, le problème de l'oppression des femmes est lié à l'identité sexuale des êtres humains et la solution consiste à éliminer les distinctions sociales entre les sexes au profit d'êtres androgynes. Pour le féminisme radical technophile de Shulamith Firestone ${ }^{11}$, l'oppression des femmes repose sur un fondement biologique, leur faiblesse physique due à la physiologie de la reproduction, laquelle, liée à l'impuissance totale des nouvelles-nées, a placé femmes et enfants sous la dépendance de l'homme, créant ainsi la première division entre classes sociales et le fondement de toutes les autres formes de domination; la solution réside dans la socialisa. tion de la procréation et de l'éducation des enfants grâce à des procédés fiables de contraception et à la gestation extra-utérine. Pour le féminisme radical gynophile, le problème découle de la biologie masculine, plus précisément, du rapport entre agressivité et testostérone, par opposition aux qualités nurturantes ${ }^{12}$ résultant du fait que seule la femme enfante; l'idéal, dès lors, n'est plus l'androgyne, parce qu'il ne s'agit pas de mélanger masculinité et féminité, mais au contraire de permettre le plein épanouissement des pouvoirs humains de la femme, dont la fonction reproductrice et les caractéristiques psychologiques spécifiques ont une valeur spéciale. Enfin, pour le féminisme

9. Sur la description du féminisme radical, cf. p. 11-12, 84-105, 249-286.

10. Ces conceptions sont désignées par les expressions * sex roles and androgyny *, *women's biology as the problem *, "women's biology as the solution *, *one is not born a woman *. Si je me permets d'utiliser des étiquettes différentes, c'est parce que ces expressions ne sont guère utilisables en tant qu'étiquettes, sauf à s'accommoder de circonlocutions comme : cette variété du féminisme radical selon laquelle on ne naît pas femme*.

11. Jaggar présente dans ce contexte deux autres auteures; mais comme elle déclare que l'une (Sherfey) n'est pas féministe et que I'autre (Brownmiller) est plutor libérale, la seule véritable représentante radicale de cette sous-tendance ne peut etre que Firestone.

12. Cf. Hurtig et Pichevin (1986:19) : «Enfin, ce n'est pas par un gout prononcé des anglicismes ou du franglais que nous avons conservé le terme de * nurture * pour le NURTURE anglais, passé dans les habitudes langagières des psychologues, méme si certains preferent opposer à nature le terme de *culture*. Nurture comme culture désignent l'influence du milieu par opposition à l'apport héréditaire, opposition que l'on retrouve dans le couple innéacquis. Mais le terme de «nurture», plus imagé, nous semble plus propre à rendre compte de la totalité des apports du milieu, dont l'individu est nourri et se nourrit; lait de la nourrice comme langue maternelle participent de la nurture.. 
radical asexuel ${ }^{13}$, le sexe lui-même est une création sociale procédant de l'élimination de toutes les caractéristiques transsexuelles de l'humanité; par suite, si l'homme et la femme sont des catégories politico-économiques, il est vain de réclamer une société matriarcale ou de promouvoir le séparatisme, l'idéal est une sorte d'androgynie non psychologique, une société asexuée.

Le problème est donc le suivant: si les tendances du féminisme se caractérisent chacune par une conception irréductible de la nature humaine, comment peut-on admettre l'existence d'une tendance radicale véhiculant quatre théories différentes de l'être humain, et si la typologie doit éviter la prolifération des sous-tendances au profit des tendances générales, comment peut-on articuler le féminisme radical en quatre orientations? Une réponse partielle à ce problème provient des caractéristiques communes à ces sous-tendances: selon Jaggar, elles reconnaissent toutes que l'oppression des femmes est fondamentale et irréductible ; que la domination masculine repose sur le contrôle des capacités sexuelles et reproductrices des femmes, qui transforme celles-ci en mères forcées et esclaves sexuelles; que les institutions par lesquelles s'exerce ce contrôle, par exemple l'éducation des enfants, le mariage, le travail domestique, la prostitution, le viol et l'hétérosexualité, ne relèvent pas de la sphère "personnelle» mais doivent être analysées politiquement; et enfin que la lutte séparatiste pour la libération des femmes, c'est-à-dire pour leur autodétermination sexuelle et reproductrice, est prioritaire et préalable au but poursuivi à plus long terme : « radical feminism seeks to build a womanculture, a new society informed by the radical feminist values of wholeness, trust and nurturance, of sensuality, joy and wildness» (p. 270). Mais comme ces caractéristiques communes ont toutes trait à l'analyse de l'oppression et à la vision de la libération, n'en faut-il pas conclure que les facteurs constitutifs d'une tendance du féminisme sont indépendants des conceptions de la nature humaine, lesquelles peuvent tout au plus servir de point de départ explicite ou implicite à l'analyse de l'oppression ? Car si la présentation des quatre conceptions radicales de la nature humaine semble suggérer quatre formes d'analyse de l'oppression et quatre modèles de libération, les chapitres

13. Jaggar se rêfere ici surtout aux féministes françaises (Delphy, Cixous, Irigaray, Wirtig) qui se réclament du matérialisme; mais comme elle prétend par ailleurs que les trois dernières sous- tendances du féminisme radical sont matérialistes, il serait peu éclairant d'assigner cette caractéristique à la présente sous-tendance. 
ultérieurs consacrés à l'analyse de l'oppression et à l'esquisse de la nouvelle société offrent une vision unifiée par rapport à laquelle les sous-tendances ne sont pas articulées. Cette articulation n'irait d'ailleurs pas de soi. Dans la mesure en effet où le féminisme radical technophile suggère un idéal androgyne, on peut se demander en quoi il differe du féminisme radical androgynophile ${ }^{14}$, et cela d'autant plus que le premier n'identifie pas l'homme comme étant l'ennemi et, en ce sens, s'accorde mal avec le séparatisme croissant de la tendance, alors que le second est lui aussi accusé de se détourner des formes spécifiques de combat contre l'homme qui exigent séparatisme et polarisation des sexes. En fait, si le séparatisme est une caractéristique du féminisme radical, il faudrait éventuellement conclure que ces deux sous-tendances, en plus d'être difficilement distinguables, ne relèvent pas ou ne relèvent plus du radicalisme. D'autre part, si le féminisme radical asexuel promeut lui aussi une forme d'androgynie, on peut soutenir que les quatre sous-tendances ne présentent que deux modèles de libération : l'androgynie et le matriarcat.

Le second problème d'inclusion a trait au féminisme socialiste. Dans la première étude de Jaggar, nous avons constaté que celui-ci n'était peut-être, selon l'auteure, qu'une extension du féminisme marxiste classique, même s'il se présentait comme une correction de celui-ci grâce au féminisme radical. L'ouvrage de 1983 permet-il de lever cette ambiguïté ? L'auteure reconnaît d'emblée ${ }^{15}$ que ce paradigme est peut-être le plus discutable des quatre. D'une part, en effet, il est peut-être impossible de le séparer clairement des formes les plus récentes du féminisme radical, dont il partage la conviction que la domination exercée par l'entremise de l'identité sexuale est aussi fondamentale que celle qui s'exerce par la classe économique. D'autre part, on peut le considérer comme une révision mineure du marxisme traditionnel. Mais, selon Jaggar, il n'est réductible ni au féminisme radical, dont les versions les plus populaires sont engluées dans l'idéalisme ou le biologisme, ni au marxisme, dont il révise fondamentalement les catégories. De l'un et l'autre, il constitue en fait une synthèse délestée des problèmes spécifiques à chacun. Rejetant le dilemme qu'il faille s'occuper d'abord des classes (marxisme) ou priori-

14. Celui-ci, il est vrai, situe le problème de l'oppression des femmes au niveau des roles sexuaux, tandis que Firestone invoque une cause biologique : mais il n'est pas prouvé que ces deux points de vue soient incompatibles.

15. En page 12. Les autres éléments urilisés dans la discussion proviennent des pages $123-126,147,303,306,387$. 
tairement des femmes (féminisme radical), il postule que le capitalisme, la domination masculine, le racisme et l'impérialisme sont inséparables et doivent être simultanément abolis : une pleine compréhension du capitalisme exige qu'on le reconnaisse structu ré par la domination masculine, une pleine compréhension de la domination masculine requiert qu'on la reconnaisse organisée par la division capitaliste du travail ${ }^{16}$. Dans la mesure où il retient la méthode du matérialisme historique, le féminisme socialiste est pour Jaggar une forme de marxisme, et même le plus orthodoxe des marxismes. Mais si, abstraitement, sa conception de la nature humaine est celle du marxisme, elle s'en écarte toutefois en concevant différemment l'activité productrice : la domination masculine y devient une partie du fondement économique de la société et ce fondement est redéfini de façon à inclure l'activité sexuelle et l'éducation des enfants. En abolissant la distinction entre sphère privée et sphère publique, le féminisme socialiste propose une vision de la bonne société qui est plus proche de celle du féminisme radical que de celle du marxisme. Mais, contrairement aux radicales, les féministes socialistes n'excluent pas les hommes: "Since women cannot transform reality alone, they must also find ways to work politically with men without being dominated by them and men may even be able to contribute to women's theoretical work» (p. 387).

Cette description du féminisme socialiste apporte une importante précision à sa définition : il ne se contente pas, dans son analyse de l'oppression, de conjuguer le sexe et la classe, puisqu'il adjoint à ceux-ci une troisième variable, celle de la race. En ce sens, il constitue une tendance originale, et son inclusion ne pose problème que dans la mesure où l'auteure affirme que: "Socialist feminism is commited to the basic Marxist conception of human nature as created historically through the dialectical interrelation between human biology, human society and the physical environment". Si l'on admet en effet que les divergences que note ensuite Jaggar ont trait à la façon d'analyser l'oppression plutôt qu'à la conception même de l'être humain, et si l'on se rappelle que chaque tendance est censée reposer

16. Si ce postulat était vrai, la domination masculine serait impossible en régime socialiste, ce que l'auteure, bien entendu, n'admet pas par ailleurs: «Males define and control all the institutions of all * national * cultures - including every purportedly socialist nation that has ever existed * (p. 250). D'un point de vue théorique, on pourrait bien entendu objecter qu'aucun pays véritablement socialistc n'a encore jamais existé. Mais pour que cette objection devienne convaincante, il faudrait alors montrer que dans les pays qui se prétendent socialistes, la division du travail est en fait capitaliste. 
sur une conception irréductible de la nature humaine, il faudrait conclure que le féminisme socialiste est une tendance originale en termes de facteurs de l'oppression, mais une sous-tendance du féminisme marxiste en termes de conception de la nature humaine. Conclusion que l'on peut éviter en soutenant qu'une tendance se définit essentiellement par son analyse de l'oppression et son modèle de libération.

\section{Passons maintenant aux problèmes d'exclusion.}

Le premier a trait aux conceptions religieuse et existentialiste du féminisme, qui sont à peine mentionnées ${ }^{17}$ : "They are outside the mainstream of contemporary feminist theorizing, and they have little direct connection with socialist feminism, the version of feminist theory that $I$ consider the most plausible». Une telle application du critère de plausibilité équivaut à une pétition de principe. Quant à l'argument historique, il a une certaine vraisemblance dans le cas du féminisme religieux, si l'on rattache celui-ci à des militantes de la première vague du féminisme américain comme Sarah Grimké et Elizabeth Cady Stanton, mais il est d'autant plus difficile à justifier dans le cas de Simone de Beauvoir que celle-ci se réclamait expressément du socialisme :

Cependant il ne faudrait pas croire que la simple juxtaposition du droit de vote et d'un métier soit une parfaite libération : le travail aujourd'hui n'est pas la liberté. C'est seulement dans un monde socialiste que la femme en accédant à l'un s'assurerait l'autre. (1968B :432)

Si l'exclusion des féminismes religieux et existentialiste est légitime, ce n'est donc pas parce que les critères sur lesquels elle repose sont pertinents, sauf dans le cas du critère historique mais à condition qu'aucune orientation du féminisme contemporain ne puisse être considérée comme "religieuse". Pour éviter la pétition de principe, il vaudrait sans doute mieux, dès lors, souligner que le féminisme existentialiste renvoie à une typologie implicite fondée sur les courants philosophiques (existentialisme, personnalisme, marxisme, herméneutique, etc.), et que ce féminisme philosophique renvoie à son tour à une typologie disciplinaire du féminisme (philosophie, religion, sociolo-

17. Cf. p. 10. Le féminisme religieux n'est pas décrit, aucune adepte n'en est mentionnée. On lui reconnaît simplement une importance historique, tout comme au féminisme existentialiste, assimilé à la simone de Beauvoir du Deuxième Sexe sans tenir compte de l'évolution ultérieure de cette dernière vers le radicalisme (cf. Schwarzer 1984 et Zephyr 1982). 
gie, psychologie, biologie, histoire, etc.) : or une telle typologie pourrait être considérée comme équivalant à une tentative de récupération du féminisme occultant ses dimensions spécifiques de critique de l'oppression masculine et de vision d'une société meilleure.

La seconde omission est celle du féminisme «noir». Celui-ci, en fait, n'est pas vraiment omis, mais plutôt disséminé sous prétexte qu'il ne présuppose pas une théorie séparée, les féministes "noires" utilisant diverses approches: libéralisme, marxisme traditionnel, séparatisme nationaliste, socialisme, radicalisme. Quant au féminisme des autres minorités raciales, il serait surtout descriptif et comporterait peu de tentatives théoriques distinctes et englobantes. De prime abord, la dissémination semble donc d'autant plus justifiée que le féminisme "noir», en tant que dimension privilégiée du féminisme racial, c'est-à-dire fondé sur la race, s'oppose implicitement au féminisme «blanc", lequel n'est pas identifié comme tel parce qu'il est lui-même réparti parmi les diverses approches théoriques. Mais comme les théoriciennes de couleur ne sont guère mises en évidence dans ces diverses approches, il en résulte l'impression, puisque "some black feminists argue that the situation of black women can be fully understood only through a development of socialist feminist theory, a development that integrates conceptually the category of racial or ethnic identity with the categories of gender and class* (p. 11), que le féminisme socialiste est le seul havre théorique à se préoccuper du problème racial dans une perspective féministe. Ne serait-il pas préférable de reconnaître la pertinence, fût-ce du seul point de vue heuristique, d'un féminisme racial où la notion de race aurait la même importance, par rapport à l'oppression des femmes, que celle de classe dans le cas du féminisme marxiste?

Le féminisme lesbien n'est pas non plus retenu, et pour une raison analogue : il ne propose pas encore une théorie de la libération des femmes à la fois distincte et englobante. Certaines de ses intuitions sont incorporées au féminisme radical, et même au féminisme libéral, mais il est davantage discuté par les radicales parce que les lesbiennes recommandent le séparatisme comme stratégie politique et que les stratégies séparatistes découlent le plus naturellement d'une analyse féministe radicale de l'oppression. De cette remarque, il résulte que le problème central est celui du séparatisme. En tant qu'orientation sexuelle, le féminisme lesbien présupposerait en effet une typologie l'opposant au 
féminisme hétérosexuel et au féminisme bisexuel, typologie qui risquerait de créer une confusion entre orientation sexuelle et allégeance féministe. N'est-ce pas précisément pour éviter une telle confusion que Ti-Grace Atkinson (1975:152) définissait le lesbianisme politique comme un " engagement volontaire et total d'une femme envers les autres membres de sa classe * et en excluait les lesbiennes qui sont également mariées, et en ce sens collaborent avec l'ennemi, tout en y intégrant les femmes qui n'ont jamais eu de relations sexuelles avec d'autres femmes, mais qui se consacrent entièrement au Mouvement?

Le dernier problème est celui du féminisme anarchiste. Le mot "anarchisme" est si vague, selon Jaggar, que l'on peut trouver des orientations qui s'en réclament en chacune des quatre tendances : dans la suspicion libérale (surtout libertaire) envers l'État, dans l'espoir du marxisme classique que l'État finira par se dissoudre, dans les attaques du féminisme radical contre le pouvoir patriarcal dans la vie quotidienne ainsi que dans sa création de possibilités d'auto-assistance, et enfin dans les critiques que le féminisme socialiste adresse à la hiérarchie et à l'autoritarisme de la gauche. Cette diversité des conceptions que l'on peut étiqueter anarchistes rend impossible, selon Jaggar, l'identification d'une conception unique de la nature humaine et de la société ${ }^{18}$ qui n'exclurait pas un nombre substantiel de soi-disant anarchistes.

Étrange argument. Selon Emma Goldman (1972:50), en effet, le mot anarchisme désigne une philosophie politique bien précise :

ANARCHISME : la philosophie d'un nouvel ordre social fondé sur la liberté non entravée par la loi faite par l'homme; la théorie que toutes les formes de gouvernement reposent sur la violence et sont par conséquent mauvaises et dommageables, aussi bien que non nécessaires.

Dans le même sens, l'anarchisme, pour Kropotkine (1970:284)

est le nom donné à un principe ou à une théorie de la vie et de la conduite en vertu de laquelle la société est conçue sans gouvernement-l'harmonie dans une telle société résultant non de la soumission à la loi ni de l'obéissance à une autorité quelconque, mais d'ententes conclues entre les divers groupes, territoriaux et professionnels, librement constitués pour fin de production et de consommation, et aussi pour la

18. Impossibilité pourtant acceptée dans le cas du féminisme radical. 
satisfaction de l'infinie variété des besoins et aspirations d'un être civilisé.

La romancière Ursula Le Guin $(1982: 121)$ résume clairement cette conception grâce à la formule suivante : « La cible principale de l'anarchisme est l'État totalitaire (capitaliste ou socialiste); son principal thème pratico-moral est la coopération (la solidarité, l'aide mutuelle)». Se méfier libéralement de l'État ou dénoncer l'au toritarisme de la gauche, ce n'est pas abolir l'État au profit de nouvelles formes d'organisation exclusivement fondées sur la coopération volontaire. En fait, s'il s'agit vraiment de ne rien sacrifier parmi les soi-disant anarchistes, ne faudrait-il pas en user de même à l'égard des socialistes, et le socialisme ne se retrouverait-il pas lui-même, sous une forme plus ou moins diluée, dans chacune des tendances? Comme le reconnaît l'auteure (p.280) : * From its original root [...], the meaning of * anarchism " (like the meanings of "socialism" and especially of "feminism ") extends to cover a broad spectrum of ideas and practices». Dans le même contexte, Jaggar affirme que le socialisme du féminisme radical n'est pas marxiste, mais plutôt libertaire ou anarchiste, ce qui accentue les interrelations entre les tendances et compromet encore davantage la thèse que chacune d'entre elles devrait reposer sur une conception irréductible de la nature humaine.

\subsection{Bilan}

Les remarques qui précèdent suggèrent que le critère de la plausibilité des tendances commande des exclusions fondées sur une pétition de principe tandis que celui de la systématicité conduit à des inclusions et à des exclusions problématiques. La principale cause des problèmes soulevés étant le postulat de la nécessité, pour une tendance, de correspondre à une conception irréductible de la nature humaine, un premier élément de solution réside dans l'abandon de ce postulat. Abandon insuffisant. Au-delà de la description de certaines orientations effectives du féminisme et de la volonté politique de promouvoir l'une d'entre elles, il nous semble en effet primordial, en utilisant les éléments fournis par cette description, de reconstituer l'ensemble des tendances possibles.

Dans la discussion du texte initial de Jaggar, une première tentative en ce sens se fondait essentiellement sur les variables impliquées dans l'analyse de l'oppression des femmes et dans l'élaboration de leur vision de la société 
meilleure. L'opposition initiale entre transformation partielle de la société (féminisme réformateur) et transformation globale (féminisme révolutionnaire) reste pertinente. Par contre, la redescription du féminisme socialiste a mis en évidence un troisième facteur dans l'analyse de l'oppression : la race. Cela nous permet de concevoir trois formes simples de cette analyse, mettant respectivement l'accent sur le sexe ${ }^{19}$, la classe et la race, et quatre formes mixtes, conjoignant deux de ces facteurs sinon les trois. Le féminisme liant l'oppression des femmes à leur sexe est un féminisme autonome. Celui qui subordonne l'oppression sexuale à la classe ou à la race est un féminisme hétéronome : classuel dans un cas et racial dans l'autre. Quant au féminisme polynome, il correspond aux quatre formes mixtes.

En termes de libération, nous disposions d'une opposition simple entre séparatisme et non-séparatisme. L'étude de la seconde typologie de Jaggar a cependant mis en évidence d'autres sèmes distinctifs : l'androgynie, le matriarcat et l'anarchie. Mais si l'on admet que l'androgynie est la contrepartie positive du refus du séparatisme, ne peut-on en déduire que la vision matriarcale de la société nouvelle est liée au séparatisme? En ce cas, les quatre principaux modèles de libération seraient d'une part le séparatisme matriarcal, anarchiste ou non, d'autre part l'androgynie, anarchiste ou non.

Par suite, si l'on ajoute au féminisme réformateur les 28 formes de féminisme révolutionnaire engendrées par la combinaison des sept types d'analyse de l'oppression des femmes et des quatre modèles de libération, l'on obtient 29 tendances théoriques :

19. $\mathrm{Au}$ sens le plus large du terme, c'est-à-dire incluant le sexe biologique et l'identité sexuale. 


\begin{tabular}{|c|c|c|c|c|c|c|c|c|c|c|c|c|c|}
\hline \multirow[b]{3}{*}{ 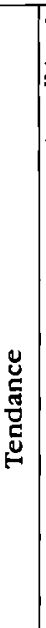 } & \multirow{3}{*}{\begin{tabular}{|l|} 
transformation \\
partielle de la \\
societé \\
(féminisme \\
réformateur)
\end{tabular}} & \multicolumn{11}{|c|}{$\begin{array}{c}\text { transformation globale } \\
\text { (féminisme révolutionnaire) }\end{array}$} & \multirow{3}{*}{$\begin{array}{l}\text { appellation } \\
\text { courante }\end{array}$} \\
\hline & & \multicolumn{7}{|c|}{$\begin{array}{c}\text { analyse } \\
\text { de l'oppression }\end{array}$} & \multicolumn{4}{|c|}{$\begin{array}{c}\text { vision } \\
\text { de libération }\end{array}$} & \\
\hline & & $\ddot{y}$ & $\begin{array}{l}\frac{\mathscr{8}}{0} \\
\frac{\pi}{0}\end{array}$ & 巳̆丶 & 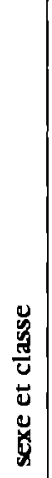 & 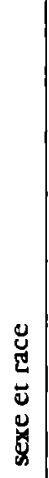 & 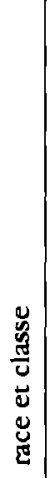 & 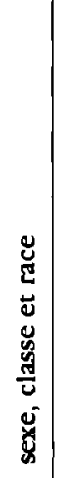 & 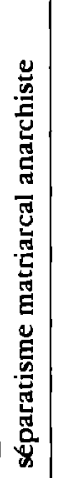 & 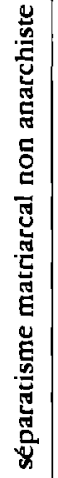 & 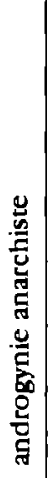 & 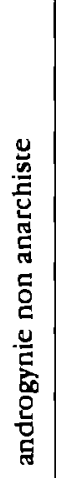 & \\
\hline 1 & + & & & & & & & & & & & & f. libéral \\
\hline 2 & & + & & & & & & & + & & & & f. radical \\
\hline 3 & & + & & & & & & & & + & & & \\
\hline 4 & & + & & & & & & & & & + & & f. radical \\
\hline 5 & & + & & & & & & & & & & + & \\
\hline 6 & & & + & & & & & & + & & & & \\
\hline 7 & & & + & & & & & & & + & & & \\
\hline 8 & & & + & & & & & & & & + & & f. marxiste \\
\hline 9 & & & + & & & & & & & & & + & f. marxiste \\
\hline 10 & & & & + & & & & & + & & & & \\
\hline 11 & & & & + & & & & & & + & & & \\
\hline 12 & & & & + & & & & & & & + & & \\
\hline 13 & & & & + & & & & & & & & + & \\
\hline 14 & & & & & + & & & & + & & & & \\
\hline 15 & & & & & + & & & & & + & & & \\
\hline 16 & & & & & + & & & & & & + & & \\
\hline 17 & & & & & + & & & & & & & + & \\
\hline 18 & & & & & & + & & & + & & & & \\
\hline 19 & & & & & & + & & & & + & & & \\
\hline 20 & & & & & & + & & & & & + & & \\
\hline 21 & & & & & & + & & & & & & + & \\
\hline 22 & & & & & & & + & & + & & & & \\
\hline 23 & & & & & & & + & & & + & & & \\
\hline 24 & & & & & & & + & & & & + & & \\
\hline 25 & & & & & & & + & & & & & + & \\
\hline 26 & & & & & & & & + & + & & & & \\
\hline 27 & & & & & & & & + & & + & & & \\
\hline 28 & & & & & & & & + & & & + & & f.socialiste \\
\hline 29 & & & & & & & & + & & & & + & f.socialiste \\
\hline
\end{tabular}


Ce tableau regroupe deux types de tendances, les unes effectives et associées à leur appellation cou rante, les autres potentielles. Ces dernières ont une portée heuristique et pourraient éventuellement permettre d'intégrer les tendances supplémentaires proposées par d'autres théoriciennes. Quant aux premières, il nous reste à préciser le sens de l'asymétrie entre féminisme réformateur et féminisme révolutionnaire, puis à expliquer la signification du dédoublement de trois d'entre elles.

Qu'une seule ligne du tableau corresponde au féminisme réformateur, cela n'implique pas que celui-ci soit moins digne d'intérêt que le féminisme révolutionnaire, mais simplement qu'au niveau d'abstraction où se situe la grille, il n'est pas utile de dresser l'inventaire de toutes les réformes possibles, parce que celles-ci ont comme point commun de ne pas affecter en principe la structure de base de la société. Des études plus poussées, et qui existent déjà d'ailleurs, pourraient, dans une perspective diachronique, rappeler comment le premier mouvement féministe américain, par opposition au mouvement contemporain, s'est surtout préoccupé de l'obtention du droit de vote pour les femmes; et, d'un point de vue synchronique, insister par exemple sur les luttes contemporaines en faveur de la libéralisation de l'avortement, de l'implantation d'un réseau de centres d'accueil pour les femmes victimes de violence masculine, d'un réseau de garderies, etc.

En ce qui a trait au féminisme révolutionnaire, les deux variétés du féminisme radical correspondent à la réduction que nous avons proposée des quatre sous-tendances décrites par Jaggar. Les deux formes de féminisme marxiste et de féminisme socialiste surprendront peut-être d'abord par leur rattachement au modèle androgyne. Dans le cas du premier, ce rattachement est opéré par Jaggar ellemême :

The major thrust, indeed, of Marxist theorizing seems to have been toward what liberal feminists have called an androgynous future $[\ldots]$ But another side to Marxist theory does emphasize the significance of the biological differences between women and men. On this view, expressed mainly in «asides * rather than in explicit argument, the biological differences between the sexes have not only determined a sexual division of labor in the past, but mean that the future can never be totally androgynous. (p. 67-68)

La vision féministe marxiste est donc androgyne, même si elle ne l'est pas totalement. Étant donné les critiques que 
Jaggar formule par ailleurs à l'égard de l'androgynie ${ }^{20}$, on pourrait penser qu'il n'en va pas de même du féminisme socialiste. Mais ce serait oublier que le concept d'androgynie est polymorphe, comme en témoigne le fait que Jaggar en discute à propos de chacune des tendances en un sens qui n'est pas toujours univoque, et que prétendre que les notions de masculinité et de féminité sont aliénantes et doivent être abolies ${ }^{21}$, c'est promouvoir une vision socialiste de la société future analogue à la société androgyne présentée par Mary Daly ${ }^{22}$ comme une société permettant la complétude psychologique et sociale des personnes, un devenir humain transcendant la polarité mutilante du masculin et du féminin. Quant à la scission des deux tendances en orientations anarchiste et non anarchiste, elle correspond d'une part à la conception marxiste d'une société où l'État, provisoirement nécessaire, finit par disparaître, d'autre part au fait que Jaggar ne thématise pas la position du féminisme socialiste à l'égard de l'anarchie.

Tentons maintenant de mettre à l'épreuve ce schème en le confrontant à un échantillon de textes mettant en cause les tendances, orientations ou courants du féminisme.

\section{Les tendances: approche polyphonique}

Selon Lucia Valeska (1981), le mouvement féministe comporte un spectre quasi infini d'orientations politiques parce que, pour en faire partie, il suffit d'être une femme. Dans une étude du féminisme américain publiée au début des années 1970, Rolande Ballorain (1972:350), dénombre 207 groupes rattachés au Mouvement de Libération des Femmes, ainsi que 110 groupes réformistes. Quant à Andrée Michel (1980), son histoire du féminisme distingue, au XXe siècle, le féminisme existentialiste de Simone de Beauvoir, le féminisme personnaliste de Betty Friedan et de la National Organization for Women, ainsi qu'un féminisme plus radical rassemblant des auteures comme Ti-Grace Atkinson et Christine Dupont. Plusieurs théoriciennes, Christine Delphy par exemple, échappent à cette ébauche de classification, dont les catégories sont par ailleurs problématiques. Ainsi, la philosophie de la National Organization for Women est assimilée au personnalisme parce qu'elle repose

20. Cf. p. 85-88. Pour une discussion génerale des critiques encourues par le concept d'androgynie : Bouchard 1989.

21. Cf. p. $316,317,327,330,340$.

22. Daly $1985: 15,21,26$ et passim. 
sur l'idée que les femmes sont d'abord des êtres humains qui, comme les autres personnes de notre société, doivent avoir l'occasion de développer à fond leur potentiel humain. Mais lorsque le féminisme existentialiste de Simone de Beauvoir est situé «dans la lignée de la philosophie personnaliste (p. 96)», on peut se demander en quoi il se distingue de celui de Friedan. Interrogation réitérée lorsqu'on lit ensuite (p. 112) que * le dénominateur commun aux efforts des féministes de tous les pays, dans les différents domaines de leurs activités, est constitué par leur conception personnaliste - le plus souvent implicite - de la dignité de la femme $[\ldots]$ ". Si le féminisme en tant que tel est personnaliste, il va en effet de soi que cette option ne peut plus servir à caractériser l'une ou l'autre de ses tendances. Au-delà de la prolifération indéfinie des options politiques et du recensement relativement exhaustif mais nécessairement provisoire des groupes réels, cet exemple montre que l'identification des tendances majeures, qui peut n'être qu'un outil de présentation dans la perspective de l'historienne, secrète des problèmes spécifiques susceptibles d'un traitement autonome.

La façon la plus simple de comparer les études consacrées directement ou implicitement aux tendances du féminisme est de considérer d'abord le nombre de tendances qu'elles intègrent, puis les sèmes distinctifs qu'elles manipulent, les hiérarchies qu'elles instaurent et les étiquettes qu'elles proposent.

À la limite, le féminisme comporte une seule tendance. Cette position est celle d'auteures qui le présentent comme s'il s'agissait d'un mouvement homogène, alors que les orientations qu'elles lui imputent sont spécifiques. Ainsi, pour Kate Millett (1983) ${ }^{23}$, le patriarcat est la principale forme de gouvernement : bien qu'on ne puisse encore résoudre la question de son origine, et même s'il comporte d'importantes variations sur le plan historique et sur le plan local, il constitue le fondement de toutes les autres structures, sociales, politiques, économiques, le modèle de toute oppression; le nouveau mouvement féministe devrait en conséquence former une coalition radicale avec les autres groupes lésés afin de réaliser la première véritable révolu-

23. L'unité sur laquelle se fonde la recherche est le document (article ou livre) plutor que la personne. II ne s'agit pas de présenter l'ensemble de la pensée d'une auteure concernant les tendances, mais la façon dont le probleme est abordé dans un document spécifique. Une meme personne peut donc illustrer plus d'une conception. 
tion sociale, celle qui libérerait l'humanité de *la tyrannie des catégories socio-sexuelles et de l'obligation de se conformer au stéréotype sexuel ${ }^{24}$ *, celle qui permettrait "d'arracher la moitié de l'espèce humaine à sa subordination immémoriale, et, ce faisant, de l'améliorer tout entière". Une telle conception relève manifestement du féminisme radical et s'oppose implicement d'une part au féminisme libéral qui refuse la révolution sociale, d'autre part au féminisme marxiste qui pose l'existence d'un matriarcat primitif et la priorité de l'oppression économique.

Un second exemple de conception monovalente du féminisme est proposé par Ti-Grace Atkison (1975), mais cette fois sur un mode polémique. En déclarant que le féminisme est par définition radical, si bien que les deux termes peuvent être employés l'un pour l'autre ${ }^{25}$, elle disqualifie en effet implicitement le féminisme non radical. Dans les textes, où elle n'opère pas cette disqualification, sa conception devient binaire et oppose le féminisme libéral, ou conservateur, ou traditionnel, ou modéré, au féminisme radical. Le premier est décrit comme une quête de l'égalité des droits pour les femmes et les hommes, quête qui refuse de reconnaître la gravité de l'oppression des femmes et suppose que la discrimination est un accident qui se corrigera de lui-même quand il sera compris. Le second considère les femmes comme une classe politique opprimée par la classe des hommes, plus précisément, par le comportement de ceux-ci, au moyen d'institutions comme le mariage, la famille, les rapports sexuels, l'amour, la prostitution et la religion. Historiquement stable et géographiquement similaire, cette oppression n'a pas connu de changements significatifs dans le temps et l'espace 26 . Transformant en obligation politique la capacité biologique des femmes à enfanter, elle constitue le fondement de toutes les autres formes d'oppression. Le protocole de libération envisagé par Atkinson stipule que les femmes doivent s'organiser entre elles pour changer la définition du rôle féminin, se libérer de celui-ci et devenir pleinement humaines : l'objectif est une société virtuellement asexuée, où le sexe ne

24. Ce qui, précise Millett, «est aussi nécessaire que l'abolition des castes raciales et des classes économiques * (p. 394).

25. Cf. Atkinson $1975: 41 \mathrm{n} .3$.

26. Cette thèse n'est pas nécessairement incompatible avec celle de Kate Millett, qui soutient que le patriarcat a subi d'importantes variations sur les plans historique et géographique : il suffit de considérer que ces variations n'ont pas affecté l'essentiel de la domination masculine. Dans l'un comme dans l'autre cas, l'accusation d'ahistoricité formulée par Jaggar (1983:116-117) devrait sans doute être nuancée. 
constituerait plus un pivot ni sur le plan individuel, ni sur le plan social. Cette solution androgyne s'oppose expressément d'une part à l'élimination physique des hommes prônée par Valérie Solanas, d'autre part à l'idée de leur mise en tutelle pour contrôler leurs inclinations sadiques, ce qui présupposerait la supériorité morale des femmes.

Si Atkinson valorise le second terme de l'opposition entre féminisme libéral et féminisme radical, Betty Friedan (1978) inverse ce rapport. Le néo-féminisme, dit-elle, doit avoir sa propre idéologie, parce que sa révolution est unique. Son but est de libérer toutes les femmes de la discrimination en tous domaines, elles ne doivent plus être asservies à la maternité, elles doivent s'autodéterminer et devenir pleinement humaines. L'homme n'est pas l'ennemi : pris au piège de l'image de sa masculinité, il doit lui aussi être libéré. Il peut même participer à la révolution, mais en laissant les femmes diriger celle-ci. Et cette révolution doit être concrète : il ne s'agit pas de dénoncer le mariage en tant que tel, ni le fait d'avoir des enfants, mais de s'en tenir aux changements possibles dans le contexte actuel : le rêve d'un monde sans sexualité ni mariage, d'une révolution effectuée sans les hommes pour libérer les femmes, relève de la fantaisie et aboutit à l'inaction, alors que l'engagement commun envers l'égalité ne peut se réaliser que dans l'action politique.

Dans une perspective historique, les deux tendances précitées peuvent aussi être décrites de façon plus neutre. Pour Rolande Ballorain (1972) par exemple, la National Organization for Women prend la relève du Women's Rights Movement : réformiste, légaliste, cet organisme tente de réaliser l'égalité des femmes et des hommes en revendiquant pour les premières des droits et des pouvoirs dans le cadre de la société existante. Révolutionnaire, le Women's Liberation Movement a par contre pour but à long terme la libération des femmes - et des hommes - à l'égard des mythes, stéréotypes et institutions sexistes. Ce but n'est cependant pas clairement défini, la ligne politique manque, l'action cède le pas aux tentatives d'accroître la conscience politique des gens par une attaque globale frôlant l'anarchisme. Lorsqu'il s'engage dans l'action, c'est de manière réformiste. De plus, le Women's Liberation Movement est non violent et il exclut les hommes.

En reformulant l'opposition de ces deux mouvements en termes de réforme et de radicalisme, Ballorain signale l'ambiguïté de ce dernier terme, qui correspond d'une part 
aux radicales féministes issues de la gauche, d'autre part aux féministes radicales pour qui le sexisme est source de tous les maux. Cette nouvelle dichotomie peut donner lieu à une autre conception binaire des tendances où le féminisme radical est confronté soit au féminisme marxiste, soit au féminisme socialiste.

C'est du féminisme marxiste qu'Evelyn Reed (1975, 1979) se fait l'apologiste. À l'encontre des radicales, elle soutient qu'il est erroné de considérer les femmes comme constituant une caste ou une classe, car cela transforme les hommes en contre-classe, ce qui implique qu'il n'y aurait pas d'hommes dans la classe opprimée, cela constitue une diversion par rapport à la lutte des classes réelles, et cela aboutit à la séparation des hommes et des femmes, à leur lutte perpétuelle ou à l'élimination des hommes. À la suite d'Engels, Reed affirme que les femmes n'étaient pas opprimées dans la société matriarcale primitive et que c'est l'avènement d'une société de classes fondée sur la propriété privée et la famille, dont le capitalisme constitue le stade ultime, qui a entraîné la dégradation et l'oppression des femmes. L'ennemi principal est donc, auiourd'hui, le capitalisme, et les hommes qu'il opprime peuvent devenir les alliés des femmes. Celles-ci ont besoin d'un mouvement autonome pour promouvoir leur cause, mais puisque le sexisme et le racisme s'enracinent dans la propriété privée, ce n'est qu'en s'alliant à l'ensemble des opprimés qu'elles contribueront à la révolution socialiste qui constitue la base de leur libération comme de celle de la classe ouvrière.

Françoise Le Calvez (1979) endosse une position analogue, mais dans une perspective socialiste. À lui seul, dit-elle, le radicalisme féministe ne peut battre en brèche le système politique américain. On ne peut séparer la lutte pour la libération des femmes du combat plus général contre la société bourgeoise et capitaliste : «sans socialisme, pas de libération des femmes; sans libération des femmes, pas de socialisme». La spécificité du combat des femmes justifie la construction d'un mouvement massif et indépendant des autres formes d'organisation tels les partis et syndicats, mais ce mouvement féministe révolutionnaire doit devenir l'une des composantes essentielles de la crise révolu tionnaire qui détruira la société bourgeoise et patriarcale.

Aucune des conceptions que nous venons d'examiner ne se présente comme une tentative de description exhaustive des tendances du féminisme. La plupart d'entre elles sont expressément polémiques. De plus, nombre de ces 
textes remontent au début des années 1970, à une époque où certains courants majeurs ne s'étaient pas encore affirmés. Ces divers facteurs peuvent expliquer les lacunes de ces descriptions, mais c'est précisément en comparant ces conceptions entre elles et avec des formulations plus complexes que l'on peut faire surgir ces lacunes. Si l'on tente un bilan provisoire, on peut constater que l'ensemble de ces conceptions monovalentes ou binaires articule en fait trois tendances.

Pour le féminisme libéral, l'oppression des femmes réside dans la discrimination dont elles sont victimes; la cause de cette discrimination n'est pas clairement assignée, mais il est au moins évident que ce n'est pas l'homme en tant que tel qui constitue l'ennemi. La libération coïncide ici avec la fin de la discrimination en tous domaines, c'est-à-dire, en termes positifs, avec l'égalité de droits pour les femmes, leur autonomie, leur accession, dans le cadre de la société existante, à toutes les prérogatives de l'humanité. Le principal moyen préconisé par Friedan n'est pas simplement, comme le suggère Atkinson, un effort de compréhension qui ferait disparaître la discrimination comme par enchantement, mais plutôt une action politique réaliste, c'est-à-dire tenant compte de ce qui est possible, et à laquelle les hommes peuvent collaborer de façon ancillaire.

Dans le cadre du féminisme radical, l'oppression des femmes, source et cause de toutes les autres formes d'oppression, découle du pouvoir des hommes, défini comme patriarcat, comme sexisme ou comme classe antagoniste. Pour Atkinson, les femmes doivent se libérer du rôle qui leur est imposé en s'organisant entre elles de façon à devenir pleinement humaines dans une société asexuée androgyne, et non en éliminant les hommes ou en les plaçant sous tutelle. Selon Millett, cette libération n'est pas incompatible avec une coalition de tous les opprimés. D'un point de vue libéral, cette révolution reste abstraite et aboutit à l'inaction. Dans la perspective marxiste ou socialiste, elle est insuffisante à renverser le système en place et elle peut même constituer une diversion, dans la mesure où faire des hommes une classe en nemie équivaut à prétendre qu'il n'y a pas d'hommes dans la classe opprimée et aboutit, selon Evelyn Reed, au séparatisme, à la lutte perpétuelle entre les sexes ou à l'élimination des hommes.

Pour les féministes marxistes ou socialistes, un mouvement autonome des femmes est nécessaire, mais il doit s'intégrer à l'ensemble de la lutte révolutionnaire, parce que 
l'ennemi principal est soit le capitalisme(Reed), soit la collusion entre celui-ci et le patriarcat (Le Calvez).

Cette première nomenclature, où le féminisme socialiste ne se distingue pas encore nettement du féminisme marxiste, n'est pas sans problèmes. Ainsi, en qualifiant de conservateur le féminisme libéral, Atkinson introduit une confusion entre celui-ci et la conception conservatrice du rôle de la femme "naturellement " vouée à la maternité et au foyer. De même, la récupération du concept de révolution par Friedan brouille indûment la distinction entre réforme et révolution, sur laquelle nous devrons d'autant plus revenir que Ballorain souligne la participation des radicales à certaines actions réformistes. Confusion, aussi, dans l'affirmation de Reed que faire des hommes une classe antagoniste implique qu'il n'y a pas d'hommes dans la classe opprimée, comme s'il n'existait qu'une telle classe et comme si les radicales tentaient de la réserver aux femmes. Enfin, le rôle des hommes par rapport à la démarche féministe devra être situé à deux niveaux : selon qu'ils peuvent ou non participer à la lutte pour la libération, et selon leur situation dans la société nouvelle.

Minimale, cette première caractérisation des tendances permet pourtant déjà de mesurer les problèmes posés par d'autres présentations ternaires. Ainsi, dans leur introduction à un important ouvrage collectif, Gould et Wartofsky (1976) distinguent trois stratégies conflictuelles du mouvement des femmes : la focalisation sur des droits égaux et des occasions d'emploi égales pour les femmes; l'approche séparatiste du féminisme radical; et l'idée que la situation des femmes ne peut changer sans une modification révolutionnaire de l'ensemble de la société. Même si elle est volontairement elliptique, cette formulation a le désavantage d'occulter la dimension révolutionnaire du féminisme radical. À l'inverse, c'est le féminisme socialiste qui devient réformiste chez Firestone (1972: 48-58), qui n'en considère que les groupes de femmes inféodés à la gauche masculine, et qui assimile par ailleurs le féminisme libéral à une position conservatrice.

D'autres conceptions ternaires précisent la distance entre le féminisme socialiste d'une part, le féminisme marxiste et le féminisme radical d'autre part. Pour Sheila Rowbotham (1973) ${ }^{27}$ par exemple, les analyses de Marx et d'Engels sont limitées parce qu'elles ont été faites par des hommes appar-

27. Voir en particulier pp. 7.9, 77-78, 86, 126-127, 289-291 
tenant à une société bourgeoise. Définir, comme Engels, la société ancienne à partir d'une étude des sociétés primitives contemporaines, cela est inacceptable, de même que subordonner la libération des femmes à celle de la classe ouvrière. La solution n'est pourtant pas de dissocier les aspirations féministes des autres mouvements de libération en ignorant délibérément que les hommes sont eux aussi opprimés dans le monde: en partant ainsi seules à la découverte de leur moi, les femmes risquent de s'enfermer dans un particularisme d'autant plus inefficace que leurs revendications impliquent des changements sociaux impossibles sans une révolution touchant tous les aspects de la société. Pour qu'advienne une véritable société démocratique communiste, les féministes doivent, selon Rowbotham, constituer un mouvement de masse des femmes de la classe ouvrière s'insurgeant contre toutes les formes d'oppression grâce à une notion de production élargie, incluant la production des femmes dans le cadre de la famille et la production des individus par la sexualité.

Semblablement, Gisèle Halimi dénonce l'insuffisance de l'approche marxiste, qui relègue l'oppression des femmes dans la sphère privée et suppose qu'elle se résorbera d'elle-même après la révolution socialiste. Au féminisme radical, elle reproche sa conception statique de l'histoire en soulignant que le patriarcat ne cause pas toujours les mêmes dégâts : l'infanticide féminin n'est pas du même ordre que l'inégalité de représentation au Comité central ${ }^{28}$. Elle lui reproche aussi, en considérant les hommes comme une classe ennemie, de promouvoir la ségrégation, laquelle ne constituerait jamais un progrès dans les luttes de libération. Halimi préconise un féminisme socialiste indépendant, intégrant les données psycho-sexuelles et la politisation du fait personnel. S'il est impossible de mener deux batailles de front, il vaudrait mieux inverser l'ordre, car détruire le patriarcat et sa culture de domination peut, en rétroaction, entraîner la suppression de l'État socialiste autoritaire et hiérarchique. Le féminisme socialiste redonnera à chaque être, homme ou femme, l'homogénéité nécessaire au-delà des dichotomies officielles scindant le corps et l'esprit, le privé et le public. Combattant pour l'égalité des femmes dans les structures existantes, le féminisme ainsi conçu

28. En se demandant ce que peut etre une dialectique des sexes sans mouvement, Halimi, comme beaucoup de marxistes et de socialistes, néglige l'avenir : qu'il y ait eu ou non un matriarcat primitif ne change rien à la volonté de mettre fin au patriarcat. D'autre part, Millett, comme nous l'avons vu, admet en principe la différence entre les types de a dégats *. 
n'aspire pas à un monde unisexuel, il ne s'attaque pas aux hommes en tant que tels, mais aux détenteurs de pouvoir.

Dans une étude consacrée au féminisme français de 1970 à 1982, Simone de Beauvoir (1984) présente une conception ternaire différente des précédentes, dans la mesure où elle oppose le fémisme radical et le féminisme socialiste non plus au féminisme libéral, mais au féminisme culturel. Composé de petits groupes inorganisés et isolés consacrés à l'éveil de la conscience et à la discussion, le féminisme radical proclame l'autonomie absolue de la lutte féministe contre l'oppression patriarcale. Coiffant le groupe Libération des femmes et la tendance Lutte des classes $^{29}$, le féminisme socialiste, placé sous la tutelle des organisations politiques, se préoccupe de problèmes comme la défense du droit au travail et l'égalité des salaires; il est souvent accusé de réformisme, mais les enjeux qu'il défend sont importants. Quant au groupe Psychanalyse et Politique, il relève du féminisme culturel. Soutenant que les femmes ne sont pas opprimées, mais plutôt réprimées, ce groupe rejette l'idée d'une base matérielle de l'oppression et la nécessité de l'action politique pour effectuer le changement social, au profit d'un culte de la néo-féminité et d'une révolution symbolique adepte de *l'écriture du corps * et de la «pensée circulaire*; la plupart de ses membres ne sont pas féministes, certaines sont même carrément antiféministes.

Sans caractériser autrement que par son étiquette la tendance lesbienne-séparatiste, Simone de Beauvoir souligne qu'elle a contribué au schisme qui a partagé le Mouvement de libération des femmes entre le féminisme radical et le féminisme socialiste. Or le problème posé par le lesbianisme avait été abordé dès 1971, dans la perspective américaine, par Anne Koedt. Certaines lesbiennes se considéraient en effet comme des féministes radicales d'avant-garde parce qu'elles avaient rejeté les hommes ainsi que les rôles sexuaux avant même l'existence du mouvement des femmes. Mais si le féminisme radical est un combat politique pour mettre fin au sexisme, c'est-à-dire pour l'abolition totale des rôles sexuels et sociaux, le lesbianisme, en tant qu'option personnelle, n'est pas en soi féministe. Il ne le devient, selon Koedt, que s'il s'insère dans le combat d'ensemble contre la suprématie masculine. Cette

29. Pour une description plus déraillée et une histoire des groupes féministes français contemporains, cf. Albistur et Armogathe (1978: 654-673) ainsi que Garcia Guadilla (1981). 
intégration n'est pas possible dans le cadre du féminisme réformiste : n'entrevoyant qu'un partenariat égal avec les hommes, celui-ci ne s'intéresse pas aux nouvelles possibilités de relations amoureuses et sexuelles entre les femmes. Seul le féminisme radical peut incorporer le lesbianisme, mais à condition qu'il ne promeuve pas une homosexualité exclusive, car alors il faudrait admettre que le sexe du partenaire importe, et donc que l'ennemi n'est pas le rôle masculin de suprématie, mais l'homme en tant qu'être biologique. Dans cette perspective, le féminisme radical, qui se distingue globalement du féminisme réformiste, comporte donc deux sous-ensembles, dont l'un se compose des lesbiennes qui, comme les radicales non lesbiennes ou bisexuelles, œuvrent à l'abolition du sexisme.

Tout comme l'asymétrie des conceptions binaires engendrait au moins trois tendances distinctes, celle des présentations ternaires suggère un bilan provisoire de six tendances : libérale, marxiste, socialiste, radicale non lesbienne, radicale lesbienne, et culturelle. Le texte de Rowbotham permet de préciser la distinction entre féminisme marxiste et féminisme socialiste : celui- ci refuse de considérer la libération des femmes comme un sous-produit de la résolution de la lutte entre les classes économiques, il situe l'avènement d'une société démocratique communiste au terme du combat contre la triple oppression liée au sexe, à la race et à la classe. L'article d'Halimi critique lui aussi la perspective marxiste orthodoxe au nom du féminisme socialiste, mais en donnant la priorité à l'oppression sexuelle et en réclamant la réforme de la société au nom d'un égalitarisme androgyne. Cet aspect réformiste du féminisme socialiste français est également souligné par Simone de Beauvoir, qui présente en outre le féminisme culturel comme une tendance récusant la lutte politique au nom d'une révolution symbolique vouée au culte de la néo-féminité. Quant à Anne Koedt, elle précise que le lesbianisme ne devient partie intégrante du féminisme radical que lorsqu'il est un combat contre le sexisme plutôt qu'une simple solution personnelle.

Nous allons maintenant nous en tenir aux seuls documents qui présentent de nouvelles tendances ${ }^{30}$.

30. À l'exception, par exemple, des textes qui décrivent les divers groupes du féminisme français dans le cadre de l'opposition réforme us révolution (Albistur et Armogathe 1978; Marks et De Courtivron); ou de ceux qui tentent de montrer la supériorité d'une tendance, tel le féminisme socialiste, sur les autres, par exemple le féminisme marxiste et le féminisme radical (Phelps 1981), ou le féminisme radical, considéré comme une forme d'idéalisme utopique, et le féminisme liberal réformiste (Socialist Workers Party 1975). 
Aux féminismes radical, libéral et marxiste, Jean Elshtain (1981 : 201-297) ajoute le féminisme psychanalytique. Les trois premiers sont décrits de façon négative. Le féminisme radical, en particulier, est présenté comme une simple inversion de la misogynie, une régression au biologisme considérant les hommes comme essentiellement mauvais et les femmes comme d'éternelles victimes ${ }^{31}$. Le féminisme marxiste n'est pas distingué du féminisme socialiste. Quant au féminisme psychanalytique, il permet de comprendre l'individu dans la société, d'apprécier plus richement les liens entre les individus et leurs mondes sociaux dans les situations historiques particulières ou universelles ${ }^{32}$.

Pour Mary Anne Warren (1980), la distinction majeure est celle qui oppose le féminisme libéral au féminisme radical. Mais elle mentionne aussi le séparatisme lesbien, sous-tendance radicale considérant l'hétérosexualité comme une institution patriarcale à abolir; le féminisme socialiste, qui admet que patriarcat et capitalisme se renforcent, mais non qu'une analyse centrée sur les facteurs économiques suffit à expliquer la domination des hommes et à permettre de s'en libérer; ainsi que le féminisme spirituel, qui se concentre sur le développement d'une conscience religieuse explicitement féministe de manière révolutionnaire (retrouver le culte de la grande déesse) ou non révolutionnaire (améliorer le sort et le rôle religieux des femmes).

Ginette Castro (1984) oppose le féminisme égalitaire ou libéral au radicalisme. Celui-ci regroupe le féminisme radical, le lesbianisme et le féminisme socialiste. Le premier se compose d'un ensemble de petits groupes non structurés et excluant les hommes, considérés comme ennemis non en tant qu'êtres biologiques mais en tant que membres d'une classe oppressive contrôlant un système sexiste; la variante pro-femmes de cette sous-tendance n'admet pas que les hommes soient victimes de leur conditionnement et susceptibles de s'améliorer : elle considère que les femmes sont parfaites, ce qui est une sorte de chauvinisme de la féminitude. Castro ajoute que le féminisme radical s'est toujours

31. Elshtain fait valoir l'existence historique de femmes non opprimées. Il n'est pas d'emblée évident que ce fait contredise de façon irréfutable les positions radicales: parce qu'il y a diverses formes d'oppression et parce qu'on peut distinguer, comme Linda Phelps (1981), le simple pouvoir, c'est-a-dire la capacité d'obtenir d'une autre personne un comportement qu'elle n'a pas nécessairement choisi, de l'autorité, qui est un pouvoir légitime, accepté par celles et ceux qui y sont soumis.

32. Elshtain mentionne dans une note le a féminisme anarchique ״ d'Emma Goldman sans le situer par rapport aux quatre tendances qu'elle a retenues 
considéré comme socialiste et qu'il promeut une société anarchiste. Elle considère que le lesbianisme, en tant que choix politique conscient, repose sur un paradoxe : il refuse au patriarcat de définir les lesbiennes par leur option sexuelle mais, en tant que féminisme, il se définit lui-même par ce choix en considérant que l'hétérosexualité opprime les femmes et que l'hétérosexisme est une forme de sexisme qui présuppose que toute femme est liée, ou désireuse de l'être, à un homme, de sorte que le lesbianisme serait, pour les femmes, la seule garantie de liberté. Quant au radicalisme féministe, c'est-à-dire au féminisme socialiste, il implique une double allégeance à la gauche et au féminisme dans le cas des femmes blanches, qui se conjoint à la lutte de libération ethnique dans le cas des femmes non blanches; son engagement fondamental est envers le socialisme et il endosse l'analyse marxiste voyant dans le capitalisme la cause première de l'oppression des femmes ; à court terme, il est réformiste. Au-delà de l'opposition entre féminisme libéral et radicalisme, Castro perçoit cependant une possibilité de synthèse dans le féminisme de l'androgynie. Concept le plus révolutionnaire du néo-féminisme, l'androgynie est un idéal de personnalité illimitée reposant sur le refus de sexualiser les caractéristiques humaines, de lier les traits psychologiques au sexe biologique. Ce concept a des implications sociales parce que la totalisation de l'être annonce la réconciliation entre les sexes, toute personne participant à l'éventail complet de l'expérience. Tout comme l'esprit est asexué, il n'y aurait pas de natures masculine et féminine, l'identité sexuelle des individus résultant d'une forme de violence. Définir les êtres en humains, et non plus en fonction de leur sexe, telle est la base du consensus le plus large. L'androgynie présuppose l'égalité de l'homme et de la femme et annonce la nécessité que cesse un jour la séparation, car le séparatisme en tant que tel n'inquiète pas le système.

Notre dernier bilan faisait état de six tendances ou sous-tendances : libérale, marxiste, socialiste, radicale lesbienne et non lesbienne, culturelle. De celles-ci, Elshtain n'en retient que trois, les tendances libérale, marxiste et radicale, auxquelles elle préfere une approche psychanalytique permettant de comprendre la situation des individus dans la société. Warren en mentionne quatre (libérale, radicale, socialiste, lesbienne), auxquelles elle ajoute un féminisme spirituel, révolutionnaire ou non révolutionnaire. Quant à Castro, elle retient le féminisme libéral et rattache au radicalisme trois sous-tendances: le féminisme radical, 
avec sa variante gynophile et androphobe, le lesbianisme, qui définit l'oppression en termes d'hétérosexualité, et le féminisme socialiste ou radicalisme féministe; de plus, elle présente l'androgynie, avec son idéal de personnalité totale non polarisée par le masculin ou le féminin, comme une synthèse possible assurant l'égalité des femmes et des hommes. En plus de la variante gynophile du féminisme radical, il nous faudrait donc tenir également compte du féminisme psychanalytique, du féminisme spirituel (révolutionnaire ou non) et du féminisme de l'androgynie?

L'un des documents les plus systématiques consacré aux tendances féministes, celui de Descarries-Bélanger et Roy (1988), identifie trois courants de pensée majeurs dans le mouvement des femmes, ainsi qu'un courant situé à sa périphérie et deux autres dans ses marges. Tout d'abord, le féminisme égalitaire considère les rôles socialement imposés dans la division sexuelle du travail comme la source de l'inégalité et de la discrimination entre les sexes ; réformiste, il préconise l'égalité de droit et de fait pour toutes les femmes dans tous les domaines ; son modèle est le masculin.

Pour le féminisme radical par contre, la source de l'oppression est le patriarcat en tant que système socio-économico-politique d'appropriation de la classe des femmes à des fins de reproduction biologique et de production économique; refusant toute explication de l'infériorisation des femmes par des arguments biologiques ou matérialistes, le féminisme radical a pour objectif l'abolition de l'ordre patriarcal et l'instauration de nouveaux rapports sociaux faisant éclater les rôles, modèles et institutions traditionnels.

Sur cette toile de fond se détachent trois sous-tendances. Révolutionnaire, le féminisme radical matérialiste relie les mentalités, institutions et lois sexistes aux structures économiques et au patriarcat défini non comme idéologie du système capitaliste ${ }^{33}$, mais comme système de relations sociales caractérisé par des relations hiérarchiques entre les sexes, par l'appropriation de la classe des femmes par celle des hommes (sexage) ; l'objectif majeur est l'abolition du patriarcat et des rapports de sexage. Pour le féminisme radical de la spécificité, l'ordre capitaliste et l'ordre patriarcal se conjuguent dans l'oppression des femmes, qui est liée à leur responsabilité dans l'éducation des enfants et dans la division sexuelle du travail; l'objectif est dès lors

33. Selon Christine Delphy (1982), la définition du patriarcat comme idéologie «a pour but d'occulter la responsabilité des hommes dans l'oppression des femmes . 
d'abolir la maternité-institution, de créer de nouvelles pratiques pour le vécu des femmes, des espaces autonomes de réflexion et de pratiques féministes. Quant au féminisme radical lesbien, s'il ne nie pas que le patriarcat soit le principal objet de la lutte, il voit dans la contrainte à l'hétérosexualité le lieu premier de l'oppression des femmes, une norme niant l'existence d'autres types de comportements sexuels et asservissant les femmes à la fonction de reproduction ; d'où la nécessité d'une société sans oppression reconnaissant l'existence lesbienne, permettant l'harmonisation des rapports mères-filles et laissant aux femmes la possibilité de s'organiser entre elles.

Le troisième courant majeur est le féminisme de la fémelléité. Contrairement aux deux orientations précédentes, qui cherchaient à abolir la différenciation entre les sexes en uniformisant fonctions et rôles, celle-ci valorise la différence et, à l'encontre de l'ordre patriarcal, revendique, de façon plus métaphorique que matérielle, un territoire spécifique de l'expérience et du savoir-pouvoir des femmes s'exprimant autant dans la création que dans la procréation.

En périphérie du mouvement des femmes, les auteures situent le marxisme féministe, pour qui l'oppression spécifique des femmes, non universelle, est liée aux formes capitalistes de production et disparaîtra avec elles, ce qui assurera aux femmes l'égalité avec les hommes ${ }^{34}$.

Enfin, au lieu d'identifier un certain nombre de courants majeurs, quitte à signaler leurs interrelations, Maggie McFaddden (1984), pour mieux cerner l'ensemble des possibilités tout en intégrant la perspective historique et les changements d'allégeance, construit deux continuums, l'un minimaliste et l'autre maximaliste, autour des rapports entre sexe et identité sexuale, chaque continuum allant de la position la plus conservatrice à la plus radicale, c'est-à-dire de celle qui opère le moins de changements dans la société existante à celle où le changement de statut des femmes et une nouvelle conceptualisation révolutionnent la société entière. Le continuum minimaliste atténue la différence et incite à l'intégration des femmes dans les systèmes masculins. Il inclut la position qui défend les droits des femmes de façon réformiste (v.g. la National Organization for Women et le groupe Cboisir); divers types de féminisme socialiste, telles la position doctrinaire donnant la priorité à la révolu-

34. Je ne retiens pas les descriptions du séparatisme lesbien et du néo-conservatisme féminin, qui sont expressément non féministes. 
tion socialiste, les diverses solutions pour faire du travail domestique un travail productif, ainsi que les tentatives de synthèses nouvelles donnant des réponses marxistes aux questions féministes (v.g. Rowbotham, Mitchell, Delphy, Eisenstein) ${ }^{35}$; puis la position qui réclame un partage des caractéristiques sexuales traditionnelles (Gilman, Chodorow, Dinnerstein); celle qui souhaite l'abolition de l'identité sexuale et des rôles (Rubin, de Beauvoir); et celle qui veut aussi abolir la reproduction biologique au profit d'une société asexuée (Firestone, Wittig). Le continuum maximaliste accentue au contraire la disparité afin de transformer, voire d'abandonner les systèmes masculins. Au-delà de la position des "sphères séparées " soulignant l'importance d'un rôle social accru des femmes à cause de leurs vertus, rôles et attitudes spécifiques, il rassemble cinq orientations : valoriser le féminin où qu'il se trouve, même dans l'homme (Cixous, Leclerc, Irigaray); créer une sous-culture féminine, éventuellement séparatiste ou tentant de recréer l'âge d'or matriarcal; célébrer la maternité comme source des valeurs différentes des femmes et de leur supériorité (Rich, Kristeva); considérer la spécificité féminine comme une force éventuellement seule capable de sauver le monde (R. Morgan, d'Eaubonne, Griffin); réclamer la séparation complète des sexes, la liberté des femmes n'étant possible que dans le cadre de leurs propres institutions (Daly, Wittig). Entre ces deux continuums, McFadden voit une médiation dans la position des féministes de couleur, qui veulent concilier la révolution socialiste et les valeurs et caractéristiques positives de leur sexe, ainsi que dans certaines œuvres d'écrivaines comme Virginia Woolf et Julia Kristeva.

Notre bilan antérieur faisait état de neuf orientations ou sous-orientations du féminisme: libérale, marxiste, socialiste, radicale lesbienne ou non lesbienne, culturelle, psychanalytique, spirituelle, androgyne. La typologie de Descarries-Bélanger et Roy est à quelques nuances près incluse dans cette énumération : le féminisme marxiste l'est nommément, le féminisme égalitaire correspond au féminisme libéral, celui de la fémelléité au féminisme culturel, et le féminisme radical se retrouve dans les deux listes, mais sa variante non lesbienne correspond dans la seconde à deux options: le radicalisme matérialiste et celui de la

35. La liste des noms propres n'est pas exhaustive; ces theoriciennes et leurs œuvres étant en principe bien connues, je m'en tiens à leurs patronymes; au besoin, on trouvera les références pertinentes dans la bibliographie de l'article de McFadden. Qu'une meme auteure illustre plus d'une position signifie que l'unité de base est l'œuvre particulière plutot que la personne. 
spécificité. La classification de McFadden, par contre, est difficilement comparable à celles que nous avons étudiées jusqu'ici, parce qu'elle s'organise autour d'un seul axe, celui de la différence entre sexe et identité sexuale. Nous nous sommes permis de cumuler les tendances comme si cette démarche allait de soi, parce que les textes examinés, sauf les deux derniers, se contentaient de décrire diverses facettes d'une réalité complexe et fluctuante sans se préoccuper de la classification en tant que telle et de ses principes, mais l'incompatibilité entre cette démarche cumulative et l'approche de McFadden nous oblige à tirer au clair, pour terminer, les problèmes que seule une telle démarche pouvait engendrer.

\section{Bilan de l'étude des tendances}

Notre comparaison de diverses études consacrées aux tendances du féminisme fait ressortir trois types de problèmes interreliés : un problème de terminologie, un problème de description et un problème de typologie.

Le problème terminologique $a$ deux volets : l'ambiguïté et le réductionnisme.

Dans la première partie de cette étude, nous avons posé que l'univers discursif initial, celui des diverses conceptions de la femme dans la société, se compose de deux sous-ensembles : les conceptions conservatrices n'admettent pas que la situation sociale de la femme est injuste, tandis que les conceptions féministes le proclament: le renouveau du féminisme, écrit Christine Delphy (1975), a coïncidé avec l'emploi du terme *oppression». Dans ce contexte, appliquer l'adjectif "conservateur" au féminisme dit libéral ne peut engendrer, comme nous l'avons souligné au passage, que la confusion. Il en va de même de la récupération * libérale " du concept de révolution : si celui-ci désigne une transformation globale de la société, on n'éclaire pas la situation en en faisant un simple synonyme du changement social en général. Une seconde ambiguité découle de l'étiquette "féminisme égalitaire " utilisée par Castro, et par Descarries-Bélanger et Roy, à la place de l'appellation plus courante de "féminisme libéral ": elle semble suggérer que celui-ci a le monopole de la quête de l'égalité, alors que cette dernière est aussi revendiquée, selon les auteures du second document, non seulement par le féminisme marxiste, mais 
aussi par les séparatistes lesbiennes ${ }^{36}$ et même par les néoconservatrices féminines. Une autre ambiguïté majeure, soulignée au passage par Ballorain, est celle du radicalisme qui, dans l'ouvrage de Castro, englobe le féminisme radical, le lesbianisme (que d'autres considèrent comme une sousorientation du féminisme radical), et le radicalisme féministe (présenté ailleurs en termes de féminisme marxiste ou socialiste).

La seconde dimension du problème terminologique relève du réductionisme. Celui-ci est parfois indissociable de l'emploi d'un vocabulaire ambigu. Ainsi, qualifier le féminisme libéral de conservateur, c'est aussi le disqualifier : si l'on manipule une opposition simple entre conservatisme et radicalisme, et si l'on assimile le féminisme au radicalisme, les réformes préconisées par le féminisme libéral s'évanou issent, celui-ci n'est même plus un féminisme. Mais le féminisme radical peut à son tour subir le même traitement : si la révolution est considérée comme le monopole de la lutte des classes au sens traditionnel, il devient une simple manceuvre de diversion au profit de la bourgeoisie.

Le réductionisme sévit également au niveau descriptif. Par exemple, présenter le féminisme socialiste comme une simple tentative de réformer les groupes de gauche dominés par les hommes, c'est ne raconter qu'une partie ou qu'un moment de l'histoire. De même, attribuer au féminisme libéral le slogan : «Fais un homme de toi, ma fille!» - c'est verser sinon dans la caricature, du moins dans une vision non dialectique suggérant que la transformation du rôle des femmes dans la société n'affectera aucunement le comportement des hommes ${ }^{37}$. Mais le problème de description le plus flagrant est celui de la fluctuation des tendances, en particulier le féminisme socialiste dans ses rapports avec les féminismes marxiste et radical.

Dans la première partie, nous avons pu constater comment Alison Jaggar distinguait le féminisme marxiste traditionnel du féminisme socialiste. La seconde partie nous propose trois autres options. Certaines, comme Eshtain ainsi que Descarries-Bélanger et Roy, présentent le féminisme marxiste sans mentionner la tendance socialiste. À l'inverse, Warren et Castro décrivent le féminisme socialiste sans référence au féminisme marxiste, et la seconde prétend qu'il endosse l'analyse marxiste de la situation des femmes,

36. Il s'agit alors, bien entendu, de l'égalité entre les femmes.

37. Castro (1984:62) souligne au contraire que la réalisation de l'égalité sexuale intégrale pronée par le féminisme libéral entrafnerait la réorganisation la plus radicale que la société (américaine) ait connue. 
ce qui est habituellement contesté par les féministes socialistes. Quant à McFadden, elle décrit trois variantes du féminisme socialiste : une approche doctrinaire, qui semble correspondre au féminisme marxiste orthodoxe; des tentatives pour conférer au travail domestique un statut de travail productif, ce qui ressemble davantage à un problème spécifique qu'à une véritable sous-orientation autonome; et des synthèses qui donnent des réponses marxistes aux problèmes féministes. Sheila Rowbotham, une féministe socialiste, est classée dans ce dernier groupe, mais le problème est qu'elle refuse expressément de donner des réponses marxistes aux problèmes féministes et qu'elle prétend, comme les autres socialistes, que le féminisme doit repenser les problèmes sociaux à la lumière non seulement du matérialisme, mais aussi de l'oppression des femmes. La situation se complique encore du fait que certaines théoriciennes (Castro, McFadden) soutiennent que le féminisme radical n'a jamais renoncé à la révolution socialiste, et du fait que le féminisme radical matérialiste, tel que le décrivent Descarries-Bélanger et Roy, a des positions identiques à celles du féminisme socialiste.

Ce qui nous ramène à un problème de terminologie et, plus fondamentalement, au problème des principes de la classification des tendances du féminisme.

La plupart des tentatives que nous avons examinées juxtaposent un certain nombre de tendances sans mentionner de critères de sélection ou d'organisation, ce qui suscite plusieurs difficultés. Elshtain, par exemple, juxtapose les féminismes libéral, radical, marxiste et psychanalytique. Or si les trois premiers peuvent être considérés comme des orientations politiques, il n'en va pas de même du dernier, qui relève d'une autre série. Comme la biologie, la psychologie ou l'histoire, la psychanalyse est une discipline qui peut être utilisée par l'une ou l'autre des trois orientations politiques retenues par Eslhtain, ainsi qu'en témoigne son rôle dans La dialectique $d u$ sexe de la radicale Shulamith Firestone ${ }^{38}$. Pour Anne Warren, la distinction majeure est

38. Le même problème est posé par l'ouvrage de Josephine Donovan (1985), qui étudie respectivement le féminisme libéral des Lumières, le féminisme culturel, féminisme et marxisme, féminisme et freudisme, féminisme et existentialisme, le féminisme radical et la nouvelle vision morale féministe. D'un point de vue typologique, ces différents aspects ne s'opposent pas entre eux. Par exemple, pourquoi traiter séparément du féminisme radical et des rapports entre féminisme et existentialisme si, comme le prétend Rolande Ballorain (1972:373), les revendications du Women's Liberation Movement sont fondamentalement existentialistes et constituent un nouvel humanisme? 
celle qui oppose le féminisme libéral au féminisme radical : mais comment se situent, par rapport à ces deux tendances, les féminismes socialiste, lesbien et spirituel? S'agit-il de sous-tendances, ou de tendances différentes mais secondaires? D'autre part, si le féminisme spirituel se concentre sur le développement de la conscience religieuse, on peut se demander s'il constitue une véritable tendance ou s'il n'est pas plutôt une composante virtuelle d'une ou de plusieurs tendances; du moins est-ce ce que semble suggérer Charlotte Bunch (1981A) lorsqu'elle dénonce la dichotomie entre féminisme politico-économique et féminismes culturel ou spirituel au nom de leur nécessaire complémentarité.

Les deux seuls principes posés expressément par notre documentation sont le rapport à l'oppression et à la libération d'une part, et la différence, maximisée ou minimisée, entre sexe et identité sexuale d'autre part.

Ce dernier critère est pourtant problématique. Prétendre que le continuum minimaliste atténue la différence et incite à l'intégration des femmes dans les sytèmes masculins, alors que le continuum maximaliste accentue la disparité afin de transformer ou même d'abandonner ces systèmes, cela implique par exemple que la société sans classes économiques ni domination patriarcale prônée par les féministes socialistes, ou la société sans reproduction biologique préconisée par Shulamith Firestone, seraient des systèmes masculins, ce qui n'est pas du tout évident. En fait, tant le sexe que l'identité sexuale peuvent être maintenus, modifiés ou abolis, ce qui permet six options simples. Dans le cas du maintien de la différence, on peut ajouter une distinction entre dévalorisation et valorisation du féminin, ce qui porte à huit le nombre des cas pertinents. Or si l'on examine dans cette perspective le continuum minimaliste proposé par McFadden, on se rend compte que les options qu'elle intitule "révolution socialiste " et *droits des femmes " sont situées du côté du pôle conservateur, ce qui implique qu'elles se situent en deçà des théories réclamant un simple partage de l'identité sexuale, donc éventuellement du côté du maintien de la différence traditionnelle entre les sexes, ce qu'elle nie pourtant expressément. De même, toutes les options répertoriées dans le continuum maximaliste semblent des variations sur le thème du maintien de la différence avec valorisation du féminin. L'objectif de tenir compte du plus grand nombre possible de tendances est sans doute louable, mais il nous semble qu'articuler celles-ci par rapport à la seule notion de différence occulte en partie les enjeux politiques fondamentaux. 
Nous en revenons ainsi au seul autre principe invoqué dans notre documentation, celui du rapport entre oppression et libération. Descarries-Bélanger et Roy l'utilisent pour analyser les tendances existantes, mais il est également possible d'en faire un instrument heuristique permettant d'articuler le champ des options possibles.

Comme nous l'avons suggéré dans la première partie, l'opposition de base distingue le féminisme réformateur du féminisme révolutionnaire. Le premier est habituellement assimilé au féminisme libéral, mais cette assimilation est erronée. Même si Gisèle Halimi, par exemple, se réclame du féminisme socialiste, son objectif d'œuvrer à l'intérieur des structures existantes la situe clairement du côté de la réforme.Cette remarque ne préjuge évidemment pas du caractère socialiste de cette démarche, mais suggère plutôt qu'à l'intérieur du féminisme réformateur on pourrait distinguer une approche libérale d'une approche socialiste. D'autre part, comme l'a montré Charlotte Bunch (1981B), l'opposition entre réforme et révolution se situe au niveau de l'objectif final, ce qui signifie que les tendances révolutionnaires, comme en a témoigné notre documentation à propos du féminisme socialiste et du féminisme radical, peuvent à l'occasion utiliser des moyens réformistes.

À l'intérieur du féminisme révolutionnaire, la distinction entre les tendances s'effectue en termes d'analyse de l'oppression et de conceptions de la libération. Rien, dans les documents examinés, ne nous incite à modifier les sept types d'analyse de l'oppression proposés dans la première partie : l'oppression affecte les femmes en tant que sexe, en tant que membres d'une classe économique, en tant qu'appartenant à une race spécifique ou simultanément par rapport à deux de ces facteurs sinon aux trois. Féminisme autonome de l'oppression des femmes en tant que femmes par le patriarcat, féminisme hétéronome subordonnant la libération des femmes à celle des prolétaires ou des minorités raciales opprimées, féminisme polynome prônant la conjonction des luttes contre l'oppression.

Dans la première partie, nous avons identifié quatre visions de la libération engendrées par une double distinction entre séparatisme matriarcal et androgynie d'une part, anarchisme et non-anarchisme d'autre part. La seconde nous a suggéré quatre options : une société androgyne, une société où les hommes sont mis en tutelle, une société où les hommes ont été éliminés, et une sous-culture tentant de fonctionner comme si les hommes n'existaient pas. Ces 
quatre options peuvent être systématisées de la façon suivante. Dans la société féministe, les hommes sont présents ou absents. Présents soit comme partenaires à part entière dans une société androgyne égalitaire, soit comme une espèce dangereuse placée sous tutelle. Absents soit réellement, parce qu'ils auraient été éliminés, soit symboliquement, comme s'ils n'existaient pas ${ }^{39}$. Société androgyne, société gynocratique, société gynocentrique effective, société gynocentrique symbolique. La société gynocratique est par définition non anarchiste, puisqu'elle repose sur la domination des femmes. Les trois autres types de société sont soit anarchiste, soit non anarchiste ${ }^{40}$. Ce qui nous donne, au total, sept conceptions de la société nouvelle.

Chacune de ces conceptions étant combinable aux sept modalités de l'analyse de l'oppression, ce sont 49 tendances du féminisme révolutionnaire qui côtoient le féminisme réformateur. Toutefois, si l'on admet que les féminismes hétéronomes et polynomes sont incompatibles avec les modèles gynocratiques et gynocentriques parce que leur analyse de l'oppression requiert une alliance avec les hommes contre les formes non sexuales de l'oppression, le nombre des tendances révolutionnaires passe à 19 :

39 Les deux dernières modalités relèvent du separatisme. Le séparatisme dans la société féministe est à distinguer du séparatisme dans la lutte féministe. À ce dernier niveau, qui est celui de la stratégie, on rencontre trois options principales : les hommes peuvent participer à la lutte féministe s'ils en laissent la direction aux femmes; ils sont exclus du mouvement féministe mais alliés potentiels dans une coalition de tous les opprimés; ils sont exclus de toutes les phases du combat. Les descriptions qui décrètent que le féminisme radical est séparatiste négligent le fait que pour certaines radicales les hommes feront eux aussi partie de la société nouvelle.

40. Les dépossédés, d'Ursula Le Guin, nous offre l'image d'une société androgyne originellement anarchiste et presque redevenue autoritaire. Les bergeres de l'Apocalypse (Françoise d'Eaubonne) illustre une société gynocentrique réelle non anarchiste malgré ses intentions originelles, mais qui pourra peut-etre se régénérer. 
TYPOLOGIE DES TENDANCES THÉORIQUES DU FÉMINISME

\begin{tabular}{|c|c|c|c|c|c|c|c|c|c|c|c|}
\hline \multirow{3}{*}{\begin{tabular}{|l}
$\mathbf{F}$ \\
$\mathrm{r}$ \\
$\mathrm{c}$ \\
$\mathrm{p}$ \\
$\mathrm{s}$ \\
$\mathrm{l}$ \\
$\mathrm{l}$ \\
$\mathrm{l}$
\end{tabular}} & \multirow{3}{*}{\begin{tabular}{|l|}
$\begin{array}{l}\text { Féminisme } \\
\text { réformateur }\end{array}$ \\
(transformation \\
partielle de la \\
société dans \\
le sens de \\
l'égalité er de \\
l'androgynie)
\end{tabular}} & \multicolumn{10}{|c|}{ Féminisme revolutionnaire } \\
\hline & & \multicolumn{3}{|c|}{$\begin{array}{c}\text { fondement } \\
\text { de l'oppression }\end{array}$} & \multicolumn{7}{|c|}{ vision de la libération: société } \\
\hline & & 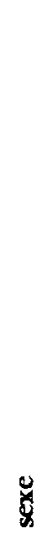 & 艺 & 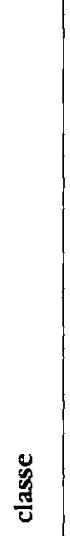 & 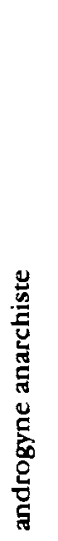 & 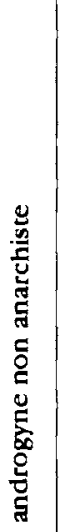 & 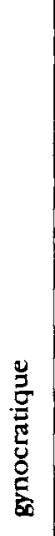 & 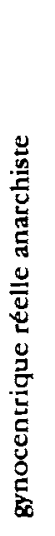 & 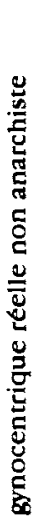 & 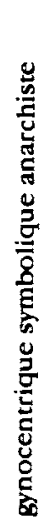 & 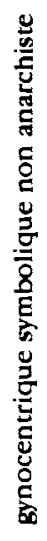 \\
\hline 1 & + & & & & & & & & & & \\
\hline 2 & & + & & & + & & & & & & \\
\hline 3 & & + & & & & + & & & & & \\
\hline 4 & & + & & & & & + & & & & \\
\hline 5 & & + & & & & & & + & & & \\
\hline 6 & & + & & & & & & & + & & \\
\hline 7 & & + & & & & & & & & + & \\
\hline 8 & & + & & & & & & & & & + \\
\hline 9 & & & + & & + & & & & & & \\
\hline 10 & & & + & & & + & & & & & \\
\hline 11$\}$ & & & & + & + & & & & & & \\
\hline 12 & & & & + & & + & & & & & \\
\hline 13 & & & + & + & + & & & & & & \\
\hline 14 & & & + & + & & + & & & & & \\
\hline 15 & & + & + & & + & & & & & & \\
\hline 16 & & + & + & & & + & & & & & \\
\hline 17 & & + & & + & + & & & & & & \\
\hline 18 & & + & & + & & + & & & & & \\
\hline 19 & & + & + & + & + & & & & & & \\
\hline 20 & & + & + & + & & + & & & & & \\
\hline
\end{tabular}


Ces 20 tendances théoriques découlent de l'articulation systématique des éléments fondamentaux fournis par la description des groupes et mouvements concrets. Si elles sont plus nombreuses que les orientations concrètes habituellement recensées alors même qu'elles s'en tiennent aux enjeux les plus généraux, c'est parce que la combinaison des éléments engendre l'ensemble des tendances possibles à ce niveau d'interprétation. Les groupes et mouvements correspondent souvent à plusieurs de ces tendances, synchroniquement ou diachroniquement, tout en conservant éventuellement le même nom, ce qui risque de leur conférer une fausse image de permanence ou, au contraire, de confusion. À l'inverse, des appellations différentes peuvent correspondre à des tendances partiellement ou complètement identiques. C'est pourquoi un catalogue des tendances possédant sa propre nomenclature peut nous aider à comprendre les mutations théoriques des entités réelles et les transformations de certains groupes. Par exemple, Gisèle Halimi et Alison Jaggar se réclament toutes deux du féminisme socialiste, mais l'orientation de la première correspond en fait à la tendance $I$, tandis que celle de la seconde correspond aux tendances 19 et $20^{41}$. De même, le tableau permet de nuancer l'affirmation que le féminisme radical n'a jamais renoncé à la révolution socialiste. Ce dernier englobe les tendances 2 à 8 , alors que le féminisme socaliste s'inscrit dans les tendances 17 à 20 , et le féminisme marxiste traditionnel, dans les tendances 11 et 12 . Si ces trois orientations avaient une même conception de la libération, elles ne pourraient différer que par leur analyse de l'oppression. Le tableau, cependant, nous montre qu'il n'en est ainsi que pour les variantes 2 et 3 du féminisme radical, et qu'en conséquence les variantes 4 à 8 sont incompatibles et avec le féminisme marxiste traditionnel et avec le féminisme socialiste. ${ }^{42}$

Un tel rôle de classification, jumelé à sa portée heuristique, permet d'espérer qu'un tel instrument n'est pas inutile.

Faculté de philosophie, Université Laval.

41. La raison de ce dédoublement a été invoquée à la fin de la première partie.

42. Le rapprochement entre certaines tendances théoriques et les courants traditionnels n'implique pas que ceux-ci soient les seules manifestations possibles de ces tendances. D'autre part, les tendances $(9-10,13-14,15-16)$ qui n'ont pas été mentionnées dans cette discussion correspondent aux diverses options accessibles à un féminisme " nationaliste ", en plus des tendances 19 et 20 , qu'il pourrait réaliser autrement que ne le font les socialistes. 


\section{BIBLIOGRAPHIE}

ATKINSON, Ti-Grace, (1975) : Odyssée d'une Amazone, Paris : Éditions des femmes.

BALLORAIN, Rolande, (1972) Le nouveau féminisme américain, Paris : Denoël/Gonthier, Coll. Femme.

BEAuvolr, Simone de, (1968) : Le deuxième sexe (2 vol.), Paris : Gallimard, Coll. Idées nos 152 et 153.

BEAUVOIR, Simone de, (1984): "France: Feminism-Alive, Well, and in Constant Danger", in MORGAN (1984), pp. 229-235.

BOUCHARD, Guy, (1989): «Cinquante-six conceptions de l'androgynie», Dialogue 28, 4, pp. 609-636.

BUNCH, Charlotte, (1981A) : «Beyond Either/Or: Feminist Options ", in QUEST (1981), pp. 44-56.

BUNCH, Charlotte, (1981B) : «The Reform Tool Kit », in QUEST 1981, p. 189-201.

CASTRO, Ginette, (1984) : Radioscopie du féminisme américain, Paris: Presses de la fondation nationale des sciences politiques.

CHOISIR, (1984) : Fini le féminisme? Paris : Gallimard, Coll. Idées, no 494.

DALY, Mary, (1985): Beyond God the Father. Toward a Philosophy of Women's Liberation, Boston: Beacon Press.

DELPHY, Christine, (1975): «Pour un féminisme matérialiste ", L'Arc, 61, p. 61-67.

DELPHY, Christine, (1982) : «Un féminisme matérialiste est possible , Nouvelles Questions féministes, 4, pp. 51-86.

DESCARRIES-BELANGER, Francine, roY, Shirley, (1988): Le mouvement des femmes et ses courants de pensée : essai de typologie, Ottawa : Les documents de l'ICREF no 19.

DONOVAN, Josephine, (1985) : Feminist Theory. The Intellectual Traditions of American Feminism, New York : Frederick Ungar.

ENGLISH, Jane, (1981) : *Introduction* à *Feminism*, in VETTERLING-BRAGGIN et al. 1981, p. 1-3. 
FIRESTONE, Shulamith, (1972) : La dialectique du sexe, Paris : Stock.

FRIEDAN, Betty, (1978) : «Our Revolution is Unique*, in MAHOWALD, (1978), p. 12-19.

GARCIA GUADILLA, Naty, (1981): Libération des femmes: le M.L.F., Paris : PUF, Le sociologue.

GOLDMaN, Emma, (1972): Red Emma Speaks, New York: Random House.

GOULD, Carol, WARTOFSKY, Marx, (éds) (1976) : Women and Philosophy. Toward a Theory of Liberation, New York : G.P. Putnam's Sons.

Halimi, Gisèle, (1984) : "Perdre plus que nos chaînes ", in CHOISIR, (1984), p. 27-45.

HURTIG, M.C., PICHEVIN, M.F. (éds), (1986) : La différence des sexes. Questions de psychologie, Paris : Éditions Tierce.

JAGGar, Alison, STRUHL, Paula R., (1978) : Feminist Frameworks, New York: McGraw-Hill.

JaGgar, Alison, (1981) : "Political Philosophies of Women's Liberation", in VETTERLING-BRAGGIN, ELLISTON, ENGLISH, p. 5-21.

JaGgar, Alison, (1983) : Feminist Politics and Human Nature, Totowa (N.J.) : Rowman \& Allanheld.

JENNESS, Linda, (éds), (1975): Feminism and Socialism, New York : Pathfinder Press.

KOEDT, Anne, (1971) : «Lesbianism and Feminism ", in KOEDT et FIRESTONE (1971), pp. 84-89.

KOEDT, Anne, FIRESTONE, Shulamith, (éds), (1971): Notes

From the Third Year, New York : Notes From the Second Year Inc.

KROPOTKIN, Peter, (1970) : Kropotkin's Revolutionary Pampblets, Ed. by Roger N. Baldwin, New York : Dover Publications.

LE CALVEZ, Françoise, (1979) : Féminisme et socialisme aux États-Unis, Paris : Union générale d'éditions, Coll. 10/18.

LE GUIN, Ursula, (1982) : The Wind's Twelve Quarters 2, New York: Granada Publishing.

MAHOWALD, Mary B., (éds), (1978) : Pbilosophy of Woman: Classical to Current Concepts, Indianapolis: Hackett Publishing Co. 
MARKs, Elaine, DE COURTIVRon, Isabelle, (éds), (1980): New French Feminisms. An Antbology, Amherst : The University of Massachusetts Press.

MCFADDEN, Maggie, (1984): «Anatomy of Difference: Toward a Classification of Feminist Theory", Women's Studies Int. Forum, 7, 6, p. 495-504.

MICHEL, Andrée, (1980): Le féminisme, Paris: P.U.F., Que sais-je? no 1782 .

MILleTT, Kate, (1983) : La politique du mâle, Paris, Coll. Points, no A56.

MORGAN, Robin, (éds), (1984): Sisterbood ls Global. The International Women's Movement Anthology, Garden City (N.Y.) : Doubleday, Anchor Press.

OKIN, Susan Moller, (1979): Women in Western Political Thought, Princeton (N.J.) : Princeton University Press.

PHELPS, Linda, (1981) : "Patriarchy and Capitalism ", in QUEST (1981), pp. 161-173.

QUEST, (1981): Building Feminist Theory, Essays from Quest, New York \& London : Longman.

REED, Evelyn, (1975) : «La biologie détermine-t-elle le destin

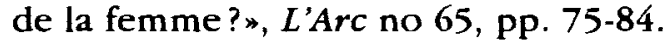

REED, Evelyn, (1979) : "Les femmes : caste, classe ou sexe opprimé», in LE CALVEZ (1979), pp. 43-57.

Rowвотнам, Sheila, (1973) : Féminisme et révolution, Paris : Petite bibliothèque Payot.

SCHWARZER, Alice, (1984) : Simone de Beauvoir aujourd'bui, Paris : Mercure de France.

SOCIALIST WORKERS PARTY, (1975) : "Towards a Mass Feminist Movement", in JENNESS (1975), pp. 129-160.

valeska, Lucia, (1981) : "The Future of Female Separatism *, in QUEST, (1981), p. 20-31.

VETTERLING-BRAGGIN, Mary, ELLISTON, Frederick, ENGLISH, Jane, (éds), (1981) : Feminism and Pbilosopby, Totowa (N.J.) : Littlefield, Adams \& Co.

WARren, Mary Anne, (1980): The Nature of Woman. An Encyclopedia and Guide to the Literature, Inverness (Calif.) : Edgepress.

ZEPHIR, Jacques, (1982): Le néoféminisme de Simone de Beauvoir, Paris : Denoël/Gonthier, Coll. Femme. 